\title{
Dust extinction and emission in a clumpy galactic disk
}

\section{An application of the radiative transfer code TRADING}

\author{
S. Bianchi \\ INAF - Istituto di Radioastronomia, Sezione di Firenze, Largo Enrico Fermi 5, 50125 Firenze, Italy \\ e-mail: sbianchi@arcetri.astro.it \\ Received 22 April 2008 / Accepted 17 July 2008 \\ ABSTRACT

\begin{abstract}
Aims. I present the Monte Carlo radiative transfer code TRADING (Transfer of RAdiation through Dust In Galaxies). The code selfconsistently computes the extinction of radiation in a dusty medium (including absorption and scattering) and the dust emission. Methods. A binary-tree adaptive grid is used for the description of the dust distribution. Dust radiation is computed at thermal equilibrium or under stochastic heating conditions, for a distribution of grains of different radii and materials. The code is applied to the case of a clumpy galactic disk, including both diffuse dust and a distribution of spherical clouds modelled on the GMCs of local galaxies. Diffuse and localised sources of starlight are used, with independent spectra.

Results. A model is provided for the edge-on galaxy NGC 891. The SED of the galaxy from the UV to the submm/mm range can be well reproduced by a bulge/disk configuration of old stars together with an extended dust disk, as suggested by the analysis of optical/near-infrared images; a clumpy dust distribution of the same mass as the diffuse dust disk, together with a UV emitting component, half of which is in the form of a diffuse disk and half in sources embedded in clouds. In total, it is found that about $35 \%$ of the bolometric radiation is absorbed (and emitted) by dust; that absorption of starlight from the old population contributes to about $60 \%$ of the dust emission. A significant component of the dust emission from clouds is due to absorption of diffuse radiation. Radial profiles of dust emission in a clumpy disk are almost independent of the wavelength, with the exception of the wavelength range on the Wien side of the thermal equilibrium peak.
\end{abstract}

Key words. ISM: dust, extinction - radiative transfer - methods: numerical - infrared: galaxies - galaxies: structure - galaxies: spiral

\section{Introduction}

The last two decades have seen works published studying the role of dust in the appearance of a spiral galaxy ${ }^{1}$. Radiative transfer models have shown that it is necessary to include scattering and consider appropriate geometries for a proper analysis of the internal extinction (Pierini et al. 2004; Tuffs et al. 2004; Rocha et al. 2008, to cite only the most recent works). Building on models of extinction in the optical, simulations of dust emission in the Far-Infrared (FIR) have been produced (Bianchi et al. 2000a; Popescu et al. 2000; Baes et al. 2004).

The comparison between models and observations has been puzzling. Dust disks of moderate optical depth can reproduce observations of edge-on spirals (Xilouris et al. 1999; Bianchi 2007), in accordance with the analysis of the extinction of background sources by less inclined disks (see, e.g., Holwerda et al. 2007). Instead, the Spectral Energy Distribution (SED) in FIR/submm requires dust absorption and emission from a significantly larger mass (Bianchi et al. 2000a; Popescu et al. 2000; Misiriotis et al. 2001).

The excess mass derived from emission models could be associated with molecular clouds (Popescu et al. 2000). Thus, it becomes necessary to study the influence of inhomogeneities, or clumping, on the radiative transfer. So far, the effects of clumping have been studied mainly in extinction for galactic disks

\footnotetext{
1 A parallel and more considerable development has occurred in radiative transfer studies of dusty circumstellar environments (see, e.g., the various models described in Pascucci et al. 2004).
}

(Bianchi et al. 2000b; Matthews \& Wood 2001; Pierini et al. 2004) and in emission for geometries appropriate for starburst galaxies (Misselt et al. 2001). In all these cases, clumps have been modelled as higher density cells in a uniformly spaced dust grid. However, the lack of resolution inside a clumpy cell does not allow us to study cases in which significant emission comes from within the cell, such as when modelling young stellar sources embedded in a molecular cloud. To obviate this, Silva et al. (1998) use a complete radiative transfer solution for a single cloud, and add the cumulative output from clouds to the dust emission from the diffuse medium. A more self-consistent solution would require the use of an adaptive mesh refinement grid, with a finer resolution for the denser medium.

I present in this paper the Monte Carlo (MC) radiative transfer code TRADING (Transfer of RAdiation through Dust IN Galaxies $^{2}$ ). For selected viewing directions, the code produces images of dust-extinguished starlight in the Ultraviolet (UV), Optical and Near Infrared (NIR), and of dust emission from the Mid-Infrared (MIR) to the submm. Images can be integrated to obtain the total SED. TRADING builds on our previous models and has been developed from the regular grid version used in Bianchi (2007).

\footnotetext{
2 The acronym was suggested by a misprint in a notice at the Exploratory Workshop: Tracing Dust In Spiral Galaxies. The workshop was funded by the European Science Foundation and took place in Ghent, Belgium on May 13-17, 2007.
} 
The scheme of TRADING is similar to that of the code DIRTY (Gordon et al. 2001; Misselt et al. 2001). However, in TRADING the dust distribution is described with an adaptive grid, while DIRTY is based on a regular grid. An adaptive grid has been included in the dust radiative transfer codes SUNRISE (Jonsson 2006), which has been used to study extinction in hydrodynamical simulations of isolated spirals (Rocha et al. 2008), and $\mathrm{ART}^{2}$ (Li et al. 2008). SUNRISE does not simulate dust emission, while $\mathrm{ART}^{2}$ includes thermal equilibrium heating from a single grain. In TRADING, instead, emission is fully taken into account by using a distribution of grain sizes and materials; it includes both thermal and stochastic heating, and self-absorption. With DIRTY, SUNRISE and ART $^{2}$ (and many other codes in the radiative transfer literature), TRADING shares the use of the MC technique, as this is easy to implement in any geometrical configuration and is not necessarily heavier in computing time than analytical techniques (Baes \& Dejonghe 2001).

TRADING has been developed, and used in this paper, to study the influence of inhomogeneities (clouds) in the dust emission from a galactic disk. To my knowledge, this is the first attempt to model the influence of dust on a galactic SED by using a fully self consistent radiative transfer calculation over scales ranging from molecular clouds to the galactic disk. Even though the code is admittedly tuned to this problem, it is still of general use and can be easily adapted to other astrophysical scenarios.

The structure of the paper is as follows. The code is described in Sect. 2. In Sect. 3, I present the implementation of the Draine \& Li (2007) dust model. In Sect. 4 TRADING is used to model the global SED of NGC 891. The results of this application, together with the main features of TRADING, are summarised in Sect. 5.

\section{The code}

TRADING consists of two main parts: the first follows the MC random walk of stellar energy packets (photons) in the dusty medium, through absorptions and scattering; a 3-D map of the Interstellar Radiation Field (ISRF) is produced and fed to the second part of the code, which computes dust emission.

The application of the MC technique to radiative transfer problems is well described in the literature. Thus, I do not provide here full details on all the algorithms used in TRADING but refer the reader to other published works (in particular, to Bianchi et al. 1996; Gordon et al. 2001; Misselt et al. 2001; Baes et al. 2003; Niccolini et al. 2003; Jonsson 2006).

\subsection{Stellar emission}

In TRADING, stellar radiation can be emitted from three different kind of distributions: an exponential disk, a spheroidal bulge and a collection of discrete spherical or point-like sources.

As usual in models of spiral galaxies, the luminosity density of the stellar disk is given by an exponential distribution along the radial, $r=\sqrt{x^{2}+y^{2}}$, and vertical, $z$, coordinates. It is

$\rho^{\text {disk }}(r, z)=\rho_{0}^{\text {disk }} \exp \left[-\frac{r}{h_{\mathrm{s}}}-\frac{|z|}{z_{\mathrm{s}}}\right]$,

where $h_{\mathrm{s}}$ and $z_{\mathrm{s}}$ are the radial and vertical scalelengths of the stellar distribution, and $\rho_{0}^{\text {disk }}$ is the central luminosity density.
The de-projection of a Sersic (1968) profile is used for the spheroidal bulge. For a profile index $n$, the luminosity density can be written as

$\rho^{\text {bulge }}(r, z)=\rho_{1}^{\text {bulge }} \frac{\exp \left[b_{n}\left(1-B^{1 / n}\right)\right]}{B^{\alpha}}$,

where $\rho_{1}^{\text {bulge }}$ is the luminosity density at $B=1$,

$B=\frac{\sqrt{r^{2}+z^{2} /(b / a)^{2}}}{R_{\mathrm{e}}}$,

$b_{n}=2 n-1 / 3-0.009876 / n, \quad \alpha=(2 n-1) / 2 n$;

$b / a$ is the minor/major axis ratio and $R_{\mathrm{e}}$ the effective radius (Prugniel \& Simien 1997). Bulges with $n=1,2,3$ or 4 are implemented in TRADING. For $n=4$ the projection of Eq. (2) on the sky plane is the $R^{1 / 4}$ (de Vaucouleurs 1959) surface brightness profile.

To simulate emission from star-forming regions within GMCs (Sect. 2.2), TRADING allows radiation also to be emitted from a list of spherical sources. Each source is characterised by its fractional luminosity relative to the total luminosity of all sources in the list, $\ell_{i}$; by its radius, $r_{i}$; and by its center in space. In the present version of TRADING, the luminosity density within each source is constant (though in this paper I mostly use point-like sources, $r_{i}=0$ ). This can be easily changed to account for a specific radial distribution. For example, the SPH smoothing kernel could be used to simulate emission from sources in hydrodynamical simulations (Jonsson 2006).

Several emission components can be included in a simulation. Each component is described by its set of parameters and by its fractional luminosity $l_{i}$ with respect to the total model luminosity. The position of emission of a photon is then derived using the MC method. First, a component $i$ is selected so that

$\sum_{k=0}^{i} l_{k}<\mathcal{R} \leq \sum_{k=0}^{i+1} l_{k}$

Throughout this paper, $\mathcal{R}$ is a random number uniformly distributed in $[0,1]$. Then, the position within the selected component is derived: the procedures to obtain the $x, y, z$ coordinates of photon emission from the exponential disk and the bulge are described in Bianchi et al. (1996) and Baes et al. (2003). To save computing time, a table with $L^{\text {bulge }}(r)$, the luminosity of a Sersic bulge emitted within radius $r$, is provided to the code, and the distance from the bulge center is obtained through interpolation.

If the photon is emitted within the list of spherical sources, the specific source first needs to be selected. This is achieved by applying Eq. (3) to the fractional luminosity of a source $\ell_{i}$. The $x, y, z$ coordinates are then extracted in a manner analogous to the bulge, with the radial distance from the source center given by $r_{i} \mathcal{R}^{1 / 3}$, as results from applying the MC method to a homogeneous sphere.

The direction of emission of photons is isotropic in the solid angle, and the initial photon energy (or weight) is set to unity. The photon wavelength $\lambda$ is obtained from the adopted stellar spectrum $F_{\lambda}$,

$\int_{\lambda_{\text {min }}^{\text {stars }}}^{\lambda} F_{\lambda} \mathrm{d} \lambda=\mathcal{R} \int_{\lambda_{\text {min }}^{\text {stars }}}^{\lambda_{\text {max }}^{\text {stars }}} F_{\lambda} \mathrm{d} \lambda$,

with $\left[\lambda_{\min }^{\text {stars }}, \lambda_{\text {max }}^{\text {stars }}\right]$ the wavelength range for stellar emission. To avoid numerical inversion of Eq. (4) within the code, a table is provided for each spectrum. Each of the emission components can have an independent stellar spectrum. 


\subsection{The dust distribution}

In radiative transfer models of spiral galaxies, dust is usually assumed to be distributed in a smooth exponential disk similar to that adopted for stars. At the reference wavelength of the $V$-band, the extinction coefficient of the smooth disk can be written as

$k_{V}(r, z)=\frac{\tau_{V}^{\text {f.o. }}}{2 z_{\mathrm{d}}} \exp \left[-\frac{r}{h_{\mathrm{d}}}-\frac{|z|}{z_{\mathrm{d}}}\right]$,

where $\tau_{V}^{\text {foo. }}$ is the central, face-on, optical depth of the dust disk, and $h_{\mathrm{d}}$ and $z_{\mathrm{d}}$ are the radial and vertical scalelengths.

If the dust properties are constant within the disk, Eq. (5) can be integrated over the volume to give a total dust mass

$M_{\text {dust }}=\int k_{V} \mathrm{~d} V\left(\frac{G / D}{m_{\mathrm{H}}} \frac{\tau_{V}}{N_{\mathrm{H}}}\right)^{-1}=2 \pi h_{\mathrm{d}}^{2} \tau_{V}^{\text {f.o. }}\left(\frac{G / D}{m_{\mathrm{H}}} \frac{\tau_{V}}{N_{\mathrm{H}}}\right)^{-1}$,

where $m_{\mathrm{H}}$ the mass of a hydrogen atom, $G / D$ is the gas $(\mathrm{H})$ to dust mass ratio and $\tau_{V} / N_{\mathrm{H}}$ is the $V$-band optical depth per unit $\mathrm{H}$ column density. The last two quantities are provided by the adopted dust model. As in Bianchi et al. (2000b), I use $\tau_{V}^{\text {f.o. }}$ as an indicator of the amount of dust in a model, regardless of whether part of the dust is distributed in clouds. That is, a dust distribution with a given value of $\tau_{V}^{\text {f.o. }}$ has the same $M_{\text {dust }}$ as a smooth model with the same central face-on optical depth.

A fraction $f_{\mathrm{c}}$ of the dust (gas) mass is assumed to be distributed in clumps, while the rest is in the smooth disk. Thus, in the presence of a clumpy distribution, the smooth disk has a central face-on optical depth $\left(1-f_{\mathrm{c}}\right) \tau_{V}^{\text {f.o. }}$. In TRADING, the clumpy distribution consists of a collection of spherical, homogeneous clouds. Following Bianchi et al. (2000b), their properties are modelled on those of Giant Molecular Clouds (GMCs).

The mass distribution of GMCs in the Milky Way and in a few other Local Group galaxies is well described by a single power law,

$$
\frac{\mathrm{d} N}{\mathrm{~d} M} \propto M^{1-\gamma},
$$

over a wide range of cloud masses (Blitz \& Williams 1999; Blitz et al. 2007). For $\gamma<2$, the $\mathrm{H}$ mass of a cloud $i$ can be derived with the MC method from

$M_{i}=\left(M_{\mathrm{inf}}^{2-\gamma}+\mathcal{R}\left(M_{\mathrm{sup}}^{2-\gamma}-M_{\mathrm{inf}}^{2-\gamma}\right)\right)^{\frac{1}{2-\gamma}}$,

with $M_{\text {inf }}$ and $M_{\text {sup }}$ the minimum and maximum mass allowed in the spectrum. The total number of clouds $N_{\mathrm{c}}$ is determined by requiring the conservation of the dust mass in the clumpy component, i.e.

$(G / D)^{-1} \sum_{i}^{N_{\mathrm{c}}} M_{i} \approx f_{\mathrm{c}} M_{\mathrm{dust}}$

(due to the random nature of the cloud mass derivation, exact equality is not assured).

Observations also suggest that the gas surface density of the clouds is independent of the cloud mass, with a mean value $\Sigma$ that changes by less than a factor of two from galaxy to galaxy (Blitz et al. 2007; Rosolowsky et al. 2003). If a constant $\Sigma$ is assumed, the radius $R$ follows directly from the cloud mass. A constant $\Sigma$ also implies a constant optical depth along a cloud diameter. For a homogeneous spherical cloud, the $V$-band extinction coefficient is then

$k_{V}=\frac{3}{4} \frac{\Sigma}{m_{\mathrm{H}} R} \frac{\tau_{V}}{N_{\mathrm{H}}}$.
As for the clouds spatial distribution, it is assumed that it follows a double exponential as in Eq. (5). The location of each cloud is also used to define the location of the spherical sources of radiation described in Sect. 2.1.

\subsection{The dust adaptive grid and the random walk}

The analytical description of the dust distribution outlined so far is used to fill up the dust adaptive grid. A 3-D binary tree (an octree) is constructed recursively subdividing each volume element (a cell) into eight children. First, the dust distribution is enclosed in a cubic volume of size $D$ (the root cell). Then, the condition

$\int_{\text {cell }} k_{V} \mathrm{~d} V \leq E \int_{\mathrm{D}^{3}} k_{V} \mathrm{~d} V$

is checked, with $E<1$ a grid refinement parameter (Wolf et al. 1999; Kurosawa \& Hillier 2001). I have used $E=10^{-5.5}$ for the simulations presented in this paper. If the condition is not met, the cell is split into eight cubes and the condition checked recursively until it is met. If the condition is met, subdivision is ended and a leaf cell is found. If $L$ subdivisions have taken place, the leaf cell has size $D /\left(2^{L}\right)^{3}$. For each cell, information on its boundaries are stored, together with pointers to its father cell (unless it is the root cell) and to its eight children (unless it is a leaf cell). The local value of $k_{V}$ is assigned to each leaf cell.

The criterion in Eq. (9) is based on the mean opacity of a cell (equivalently, on the density, if the dust properties are identical throughout space, as in this work). Since it is not tied to the local radiation field (which is not known before the radiative transfer calculations), it may fail in producing an adequate cell resolution in the presence of very strong field gradients. A high resolution is needed for the case of clouds with internal point sources, and could in principle be achieved by using a smaller value for $E$. However, this would result in a larger number of cells and in an unnecessary finer sampling of the diffuse medium ${ }^{4}$. To avoid this, the grid algorithm allows one to force splitting in cells: if the cloud diameter is smaller than the dimension of a cell of level $L$, the leaf cells can be split further by $l$ sublevels, down to level $L+l$, thus providing at least $2^{l-1}$ resolution elements along a cloud diameter. An example of the binary structure of the dust grid is shown in Fig. 1.

The optical depth of the dust distribution along any given direction is found from the intercepts of the photon path with the boundaries of the leaf cells in the grid (for more details, see Kurosawa \& Hillier 2001). The leaf cell that contains the position of emission is found by recursively ascending the binary tree from the root: the point location algorithm of Frisken \& Perry (2002) is used. The coordinates of the intercept of the photon path with the boundaries of this first cell are used to locate the next cell along the path. This neighbour cell could be found again by ascending the tree from the root. An alternative

\footnotetext{
3 As an example, let's consider a homogeneous root cube: setting, e.g., $E=8^{-3}$ is equivalent to produce a regular grid of $8^{3}$ leaf cells (descending down to level $L=3$ ), each of size $D / 8$. Using a cubic root volume to enclose a thin disk-like structure may seem inadequate, since regions distant from the galactic plane are empty. However, these regions are enclosed by a limited number of low-level leaf cells. Furthermore, the cubic geometry makes the structure of more general application and is more effective in the description of the spherical clouds in the clumpy distribution than cells with a reduced size along $z$.

${ }^{4}$ For smooth models, and for the smooth medium in the clumpy models presented here, the chosen $E$ value ensures enough spatial resolution for the convergence of the results.
} 


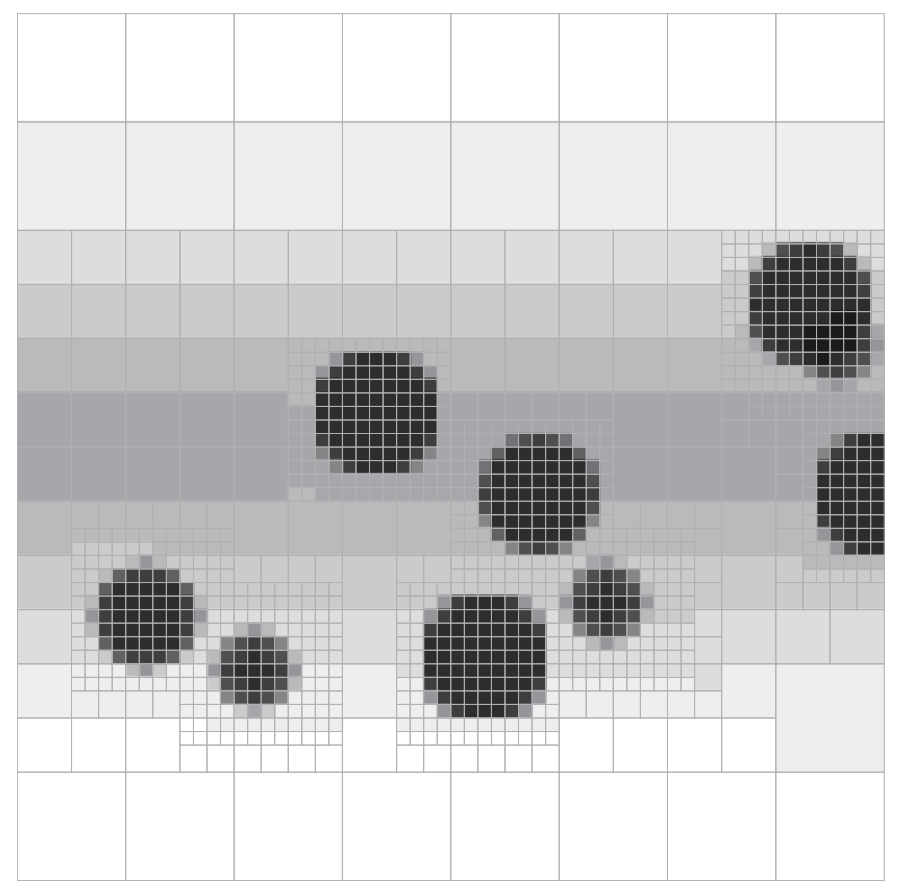

Fig. 1. A cut through the binary dust grid used in Sect. 4.2, perpendicular to the galactic disk. Since mean values are stored in each cell, the density on the border of clouds does not drop abruptly to the value of the smooth medium. The size of the box is $1.5 \mathrm{kpc} \times 1.5 \mathrm{kpc}$.

scheme would consist of finding the smallest non-leaf cell that is a common ancestor of both a cell and its neighbour: the tree is then descended from the first cell to the common ancestor, and then ascended again from the ancestor to the cell neighbor. This second implementation is more efficient (Frisken \& Perry 2002). If $\mathrm{d} l$ is the length of the intercept, the optical depth along the path in the cell is

$\mathrm{d} \tau_{\lambda}=A_{\lambda} k_{V} \mathrm{~d} l$,

where $\lambda$ is the wavelength of the photon and $A_{\lambda}$ is the dust extinction law from the adopted model. The process is then repeated and the optical depth along the path $\tau_{\lambda}$ accumulated, until the photon suffers the first scattering event (or the boundary of the root cell is reached).

The location of the scattering event is determined randomly, by finding the optical depth

$\tau=-\log (1-\mathcal{R})$,

and stopping the path in the grid where $\tau_{\lambda}=\tau$. If $\tau$ is larger than the total optical depth along the chosen direction, the photon escapes dust. To ensure that all photons contribute to the scattered flux, even in the case of low global opacity, scattering may be forced (Cashwell \& Everett 1959). Typically, the first scattering event is forced, while the following are determined according to Eq. (10) (Bianchi et al. 1996; Gordon et al. 2001). The same scheme is adopted here.

In the scattering position, the photon propagates along a new direction, which is derived randomly from the adopted scattering phase function. Here I use the Henyey \& Greenstein (1941) phase function, dependent on the scattering asymmetry parameter $g_{\lambda}=\langle\cos (\theta)\rangle$, with $\theta$ the angular deviation between the new and the old directions. The Henyey \& Greenstein phase function is a reasonably good approximation for the wavelengths considered here (Draine 2003b). The values for $\omega_{\lambda}$ and $g_{\lambda}$ are provided by the adopted dust model.
The scattered photon has its weight reduced by the albedo $\omega_{\lambda}$, while the fraction $\left(1-\omega_{\lambda}\right)$ is absorbed. The absorbed energy could be stored in the leaf cell where scattering takes place and used later to derive dust emission (Bianchi et al. 2000a; Gordon et al. 2001). However, it is more efficient to store the absorbed energy along all the cells crossed by a photon rather than at the end point only, a concept introduced by Lucy (1999) and described in Niccolini et al. (2003) for both the non-forced and forced scattering cases. Using this formalism, TRADING accumulates the amount of energy absorbed as a function of the wavelength, $W_{\lambda}$, from which the local ISRF is derived,

$J_{\lambda}=\frac{1}{4 \pi k_{\lambda}\left(1-\omega_{\lambda}\right)} \frac{W_{\lambda}}{\Delta V}$,

with $\Delta V$ the cell volume. For each leaf cell, the average of $J_{\lambda}$ is stored in $N_{\text {SED }}^{\text {stars }}$ contiguous wavelength bins, logarithmically spaced in the range $\left[\lambda_{\min }^{\text {stars }}, \lambda_{\max }^{\text {stars }}\right]$.

After the first scattering, the whole procedure is reiterated, until the photon weight falls below a limit value $\left(10^{-4}\right.$ is used here). A number $N_{\text {phot }}$ of photons are run to provide high signalto-noise images and $J_{\lambda}$ values in the grid. The model can be normalised to physical values by multiplying the outputs by $L_{\mathrm{bol}} / N_{\text {phot }}, L_{\mathrm{bol}}$ being the bolometric luminosity assumed for the stellar sources.

Simulated images of dust-extinguished starlight can be also produced, for all the $N_{\text {SED }}^{\text {stars }}$ bins or at specific values of $\lambda$. Any viewing direction is possible. This is achieved using the peelingoff technique of Yusef-Zadeh et al. (1984), in which each emission and scattering event contributes to the surface brightness distribution. The technique allows one to produce high signalto-noise results without the need to collect photons in a broad angle band (see, e.g., Bianchi et al. 1996), thus preventing image smearing when there is a strong gradient with the viewing direction (as for, e.g., a disk seen edge-on).

Once that the radiation field has been determined, it may result that some cells contribute negligibly to the total absorbed (and emitted) energy. To avoid unecessary calculation, the grid is reduced in size by adopting a criterion similar to that in Misselt et al. (2001): cells are ordered according to the amount of energy absorbed within them, and a threshold is derived so that all cells below the threshold contribute to a fraction $f_{\text {abs }}$ of the total absorbed energy. The grid is recursively accessed to find fathers of leaf cells which have all eight children below the threshold. When such cells are found, the children are cut and their amount of absorbed energy is transferred to the father, which becomes a leaf. Instead, when leaf cells below the threshold are isolated, they are excluded from the calculation of dust emission. When a clumpy dust distribution is considered, even a small value of $f_{\text {abs }}$ may result in an appreciable reduction of the memory occupation of the grid and of the execution time of the emission code. In this work, I have used $f_{\text {abs }}=0.1 \%$. No significant change in the results is produced by the adoption of this threshold.

\subsection{Dust emission}

When considering the transfer of radiation through absorption and scattering, it is not necessary to know the details on the dust composition and size distribution, but it is sufficient to consider mean properties (Wolf 2003): these are the values $A_{\lambda}, \omega_{\lambda}$ and $g_{\lambda}$ used in the previous section.

Dust emission, instead, depends on the properties of each grain. For an ensemble of spherical grains of $N_{\text {mat }}$ different materials, each characterised by a distribution $n_{k}(a)$ for the radius $a$, 
the emission coefficient (energy emitted per unit volume, time, wavelength and solid angle) for thermal equilibrium emission can be written as

$$
j_{\lambda}=k_{V} \frac{\sum_{k=1}^{N_{\text {mat }}} \int n_{k}(a) a^{2} Q_{k}^{\mathrm{abs}}(a, \lambda) B_{\lambda}\left(T_{\mathrm{d}}\right) \mathrm{d} a}{\sum_{k=1}^{N_{\text {mat }}} \int n_{k}(a) a^{2} Q_{k}^{\mathrm{ext}}(a, V) \mathrm{d} a,}
$$

with $Q_{k}^{\text {ext }}$ and $Q_{k}^{\text {abs }}$ the extinction and absorption (emission) efficiencies (i.e. the cross-sections divided by $\pi a^{2}$ ), $B_{\lambda}$ the Planck function and $T_{\mathrm{d}}$ the equilibrium temperature of a grain, dependent on the radius $a$ and on the material $k$. In TRADING, the size distribution for each material $k$ is sampled with $N_{a}^{k}$ contiguous bins logarithmically spaced between the minimum and maximum radii of the adopted grain distribution. Equation (11) thus becomes

$j_{\lambda}=k_{V} \sum_{k=1}^{N_{\text {mat }}} \sum_{j=1}^{N_{a}^{k}} Q_{j k}(\lambda) \mathcal{B}_{j k}, \quad \mathcal{B}_{j k}=B_{\lambda}\left(T_{\mathrm{d}}\left(a_{j}, k\right)\right)$,

where $Q_{j k}$ is the absorption cross section integrated over the $j$ th radius bin of width $\Delta a_{j}$, normalised by the $V$-band extinction cross section ${ }^{5}$,

$$
Q_{j k}=\frac{n_{k}\left(a_{j}\right) a_{j}^{2} Q_{k}^{\mathrm{abs}}\left(a_{j}, \lambda\right) \Delta a_{j}}{\sum_{k=1}^{N_{\mathrm{mat}}} \int n_{k}(a) a^{2} Q_{k}^{\mathrm{ext}}(a, V) \mathrm{d} a} .
$$

At thermal equilibrium, $T_{\mathrm{d}}$ can be found equating the radiation absorbed from the ISRF $J_{\lambda}$ to the dust emission. For the $j$ th grain of material $k$, the thermal balance can be written as

$$
\sum_{i=1}^{N_{\mathrm{SED}}^{\mathrm{stars}}} J_{\lambda} Q_{j k}(\lambda) \Delta \lambda_{i}=\int B_{\lambda}\left(T_{\mathrm{d}}\left(a_{j}, k\right)\right) Q_{j k}(\lambda) \mathrm{d} \lambda,
$$

where $\Delta \lambda_{i}$ is the width of the $i$ th bin used to store $J_{\lambda}$. To save computing time, the integral in Eq. (14) is tabulated, and the temperature $T_{\mathrm{d}}\left(a_{j}, k\right)$ is found by interpolation.

For small grains ( $a \lesssim 0.01 \mu \mathrm{m}$ ) heating is stochastic and it is not possible to define an equilibrium temperature. Instead, a given grain is characterised by a temperature distribution $P(T)$, dependent on the grain characteristics and on the heating field. Following the method of Guhathakurta \& Draine (1989), $P(T)$ can be derived from the transition matrix $A_{f, i}$, that gives the probability per unit time that a dust grain is heated (or cooled) from the enthalpy state $H_{i}$ to the enthalpy state $H_{f}$. In the notation used so far, for a given grain, the matrix elements due to heating by the ISRF are $(f>i)$

$A_{f, i} \propto J_{\lambda} \frac{Q_{j k}(\lambda) \Delta H_{f}}{\left(H_{f}-H_{i}\right)^{3}}$,

where $\Delta H_{f}$ is the width of the $f$ enthalpy state, $\lambda=h c /\left(H_{f}-H_{i}\right)$ is the wavelength associated with the transition, and the value of $J_{\lambda}$ is obtained by interpolating the ISRF provided by the radiative transfer code (see Misselt et al. 2001, for a full description

\footnotetext{
5 A table with $Q_{j k}(\lambda)$ is computed from the adopted dust model and provided to the radiative transfer code. Ancillary tables are produced by averaging $Q_{j k}$ over the wavelength bins used for the sampling of $j_{\lambda}$ (Eq. (12)), $J_{\lambda}$ (Eq. (14)) and $J_{\lambda}^{\text {s.a. }}$.
}

of the matrix elements). Considering only cooling terms from level $f+1$ to level $f$ (the thermal continuous cooling approximation; Draine \& Li 2001), it is

$A_{f, f+1} \propto \frac{\int Q_{j k}(\lambda) B_{\lambda}\left(T_{f+1}\right) \mathrm{d} \lambda}{H_{f+1}-H_{f}}$

where $T_{f+1}$ is the temperature associated with state $f+1$. In TRADING, a temperatures range for $P\left(T_{\mathrm{d}}\right)$ is selected, and $N_{T}$ states are defined, logarithmically spaced in temperature. The enthalpy associated with each state is derived by integrating the specific heat adopted for the grain materials over the temperature. The same temperature range and $N_{T}$ are used for all grains, regardless of the local ISRF intensity or spectrum. This allows to compute tables for the cooling terms and for the multiplicator of $J_{\lambda}$ in the heating terms beforehand, thus saving computing time. For a grain of material $k$ and radius $a_{j}$ that undergoes stochastic heating, the $\mathcal{B}_{j k}$ term in Eq. (12) becomes

$\mathcal{B}_{j k}=\sum_{t=1}^{N_{T}} P_{t} B_{\lambda}\left(T_{t}\right)$

In each cell, the emission coefficient $j_{\lambda}$ is computed by summing the contribution of all sizes and materials in the dust distribution. The calculation is carried out for $N_{\mathrm{SED}}^{\text {dust }}$ contiguous wavelength bins, logarithmically spaced in the range $\left[\lambda_{\min }^{\text {dust }}, \lambda_{\max }^{\text {dust }}\right]$.

Subsequent calculations are carried out using a no-scattering $\mathrm{MC}$ radiative transfer procedure. The $\mathrm{MC}$ allows us to take into account self-absorption, i.e. the possibility that dust-emitted radiation is absorbed again by dust. This could be relevant in some astrophysical situations, such as when MIR radiation travels through a very dense dusty medium.

In principle, the cell where a photon (of unit weight) is emitted should be selected randomly from the amount of energy emitted within it, and the wavelength determined randomly from the local $j_{\lambda}$ spectrum. However, it is not straightforward (or efficient in terms of computing time) to access the binary tree in this way. Furthermore, the procedure would also require us to store $j_{\lambda}$ in the memory for each cell. Also, since scattering is not included, calculations can be carried out at once for all wavelengths. Thus in TRADING, once $j_{\lambda}$ is derived, a few polychromatic photons are emitted (five are sufficient for the simulations presented in this paper), with a $\lambda$-dependent weight proportional to $j_{\lambda} \Delta V$ ( $\Delta V$ is the cell volume). The emission coordinates within a cubic (homogeneous) leaf cell and the direction propagation are determined in the usual way. As the photon traverses

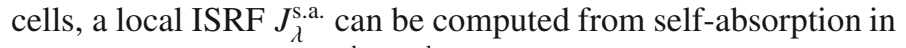
the wavelength range $\left[\lambda_{\min }^{\text {dust }}, \lambda_{\max }^{\text {dust }}\right]$, analogously to what done for stellar photons. Here, the wavelength range could be limited by a maximum wavelength $\lambda_{\text {max }}^{\text {s.a. }}$, if self-absorption is insignificant for $\lambda>\lambda_{\max }^{\text {s.a. }}$, and a different number of bins over which $J_{\lambda}^{\text {s.a. is }}$ stored, $N_{\mathrm{SED}}^{\mathrm{s} . \mathrm{a}}$, can be selected. Selecting a restricted wavelength range for self-absorption may become necessary when computer memory is a concern, since, contrary to $j_{\lambda}$, it is necessary to

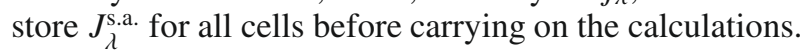

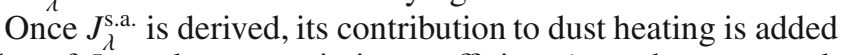
to that of $J_{\lambda}$, and a new emission coefficient $j_{\lambda}$ can be computed. The entire process is repeated until the total output of dust emission (i.e. the dusty SED integrated over the solid angle) does not change by more than a given fraction, typically a few percent. The convergence check, as well as the splitting of TRADING in the extinction and emission parts, could be avoided by using the immediate re-emission concept of Bjorkman \& Wood (2001). 


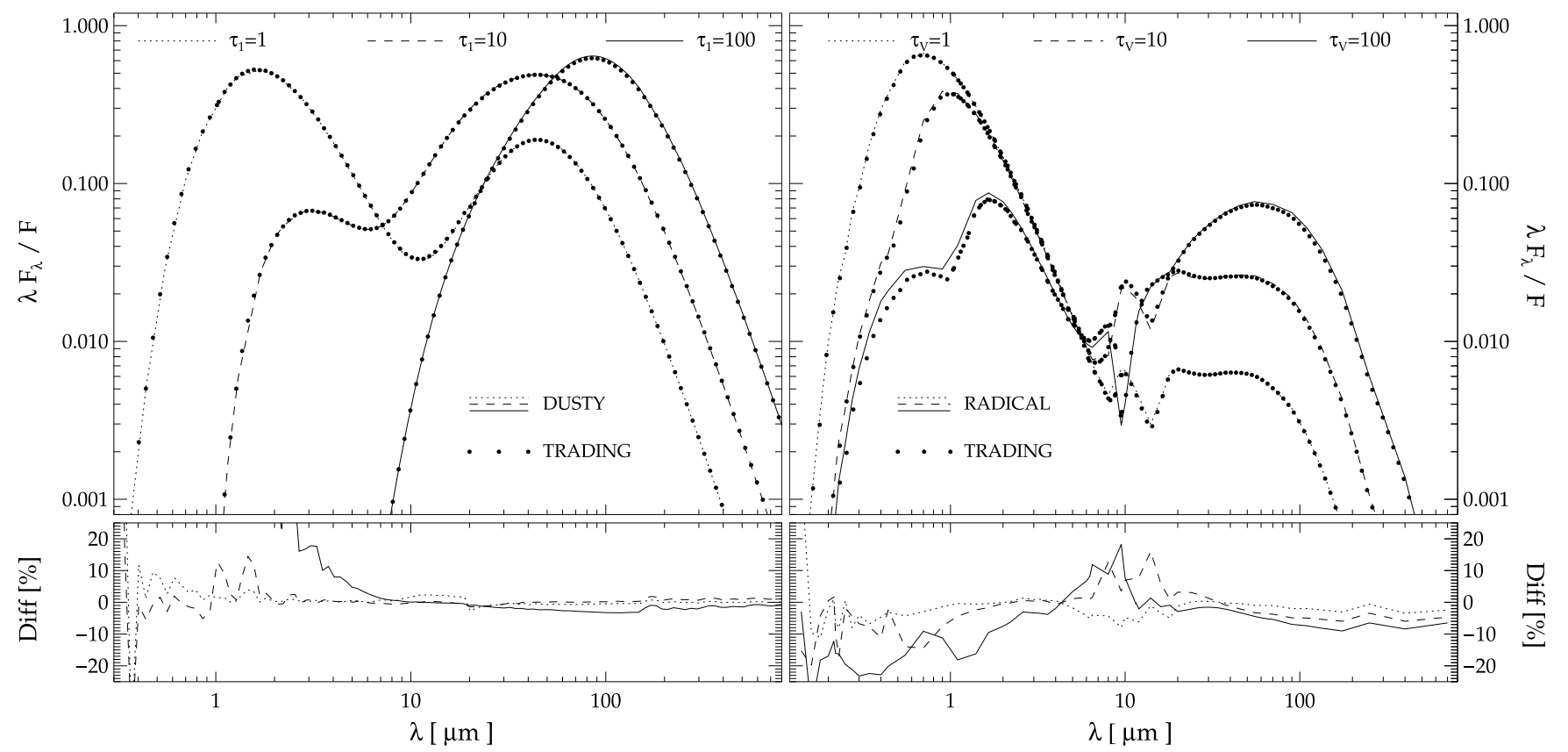

Fig. 2. Tests of the TRADING code. On the left, comparison with the DUSTY solutions of the benchmark problems of Ivezic et al. (1997) for a homogeneous sphere. On the right, comparison with the RADICAL solutions for the benchmark problems of Pascucci et al. (2004). In all cases, the SED has been normalised to the total luminosity; the relative difference has been computed with respect to the benchmark solutions. See text for details.

With this procedure, the absorption of a stellar photon is immediately followed by the emission of an infrared photon from dust, with the wavelength sampled from a distribution that corrects itself at each absorption event; eventually the distribution of infrared photons converges to the correct distribution, without the need for a separate calculation of the absorption and emission processes. Although the method has a solid mathematical grounding in the case of thermal equilibrium heating and in MC procedures where photons have weights, it is not clear yet if it can be applied with the Lucy (1999) estimator for $J_{\lambda}$ or in the case of stochastic heating (Baes et al. 2005; but see Jonsson 2006, for a different view on the second issue). Because of this, I have preferred the current scheme.

As for starlight, images of dust-emitted radiation can be obtained from any viewing direction, for all the $N_{\text {SED }}^{\text {dust }}$ bins or at specific values of $\lambda$, using the peeling-off technique from each emission point in the MC procedure.

\subsection{Benchmarking}

The code results have been tested on a few radiative transfer solutions: the outputs from the MC radiative transfer code of Bianchi et al. (1996); the description of the radiation field due to a point source in the center of a finite scattering sphere (Siewert \& Maiorino 1979); the benchmark problems in 1D geometry of Ivezic et al. (1997); the benchmark problems in 2D geometry of Pascucci et al. (2004). I describe here the comparison with the last two benchmark cases.

Ivezic et al. (1997) describe a set of radiative transfer problems for a spherical dust distribution heated by a central point source. I have compared the output of TRADING with the case of a homogeneous sphere. The solutions have been obtained in numerical format using the publicly available radiative transfer code DUSTY ${ }^{6}$, based on the method of Ivezic \& Elitzur (1997). DUSTY is one of the radiative transfer codes used in Ivezic et al. (1997).

A grid for a homogeneous sphere of radius $R_{\text {out }}$ was first constructed. The dust distribution of the benchmark cases has a inner cavity for $R_{\text {in }}=R_{\text {out }} / 1000$. Most of the sphere volume is sampled with cells of level $L=7$ (thus, having a size of $2 \times R_{\text {out }} / 128$ ), but finer subdivisions (down to $L=14$ ) are used for a proper rendering of the inner and outer boundaries. In total, the grid consists of $6 \times 10^{5}$ leaf cells. The extinction coefficient (at the reference wavelength of $1 \mu \mathrm{m}$ ) is given by the optical depth of the model along the radius, $k_{1}=\tau_{1} /\left(R_{\text {out }}-R_{\text {in }}\right)$.

DUSTY only deals with a single grain of mean properties and the scattering is assumed to be isotropic. Thus, $g_{\lambda}=0$ has been set in TRADING, and $A_{\lambda}$ and $\omega_{\lambda}$ have been chosen as in Ivezic \& Elitzur (1997).

In the benchmark cases, radiation comes from a central point source, emitting as a blackbody at $T_{\star}=2500 \mathrm{~K}$. That ISRF has been sampled with 50 bins in the range $[0.3,20] \mu \mathrm{m}$. DUSTY solutions are scale-free (Ivezic \& Elitzur 1997), and it is not necessary to provide the absolute luminosity. Instead, for the comparison with TRADING, a physical value for $R_{\text {out }}$ was assumed, and the bolometric luminosity $L_{\mathrm{bol}}$ was derived with the relations provided in Ivezic \& Elitzur (1997) and Ivezic et al. (1997). Dust radiation has been sampled with 100 bins in the range $[1,1000] \mu \mathrm{m}$. The same sampling has been used for the contribution of self-absorption to the ISRF. A total of $10^{6}$ photons have been used.

The result from the comparison is shown in the left panel of Fig. 2 for the cases $\tau_{1}=1,10$ and 100. As TRADING deals with extinction and emission separately, the stellar and dust SEDs have been summed together in the overlapping wavelength range. The inclusion of self-absorption has been necessary for

\footnotetext{
${ }^{6}$ http://www $\cdot$ pa.uky.edu/ moshe/dusty/
} 
a correct solution of the two high optical depth cases: convergence to $0.5 \%$ was required. The agreement with the benchmark cases is satisfactory, with TRADING differing from DUSTY by only a few percents in most cases. The agreement is worse at shorter wavelengths, for several reasons: the intrinsic noise in the MC procedure; the difficulty of properly sampling the integral of a steeply rising spectrum (Eq. (4)) with tables; the lack of ad-hoc optimisations for high optical depths (see, e.g., Juvela 2005). In particular, TRADING diverges significantly from the DUSTY solution for the $\tau_{1}=100$ case, but only for $\lambda \lesssim 3 \mu \mathrm{m}$ and very low flux levels $\left(\lesssim 10^{-7}\right.$ in the units of Fig. 2). Calculations for these low fluxes in the higher optical depth case also pose problems for DUSTY: numerical errors had to be prevented by requiring a significantly higher numerical accuracy than in the other cases. Nevertheless, the radiation field appears to be computed correcly by TRADING in all cases, as shown by the bulk of dust emission in the SED. Also, the surface brightness profiles of stellar and dust radiation show a similar agreement.

The geometry analysed so far is equivalent to what will be used in Sect. 4.2 for embedded stellar sources at the center of GMCs. Unfortunately, in simulations of galactic disks it is not possible to have, for each cloud, the same level of spatial resolution as in the comparison presented here. Thus, it will become necessary to check for the convergence of the simulation outputs.

A benchmark problem with a more compelling geometry is provided by Pascucci et al. (2004). They use an axially symmetric dust disk, extending from an inner cavity of 1 AU to an external boundary of $1000 \mathrm{AU}$, with a steeply rising density gradient close to the center. The dust grid I have used for this problem has been obtained with the procedure described in Sect. 2.3, but forcing cell splitting in the inner $50 \mathrm{AU}$ down to $L=14$ close to the center. The grid consists of $1.1 \times 10^{6}$ leaf cells. Dust properties are provided by the authors, and isotropic scattering is assumed.

As for the previous case, the heating source is point like and located in the center, with $L_{\text {bol }}=1 L_{\odot}$ and blackbody emission at $T_{\star}=5800 \mathrm{~K}$. For this case, the ISRF has been sampled with 50 bins in the range $[0.12,10] \mu \mathrm{m}$. All the other parameters are the same as for the comparison with DUSTY.

As a reference, I have used the solution of the benchmark problems provided by RADICAL (Dullemond \& Turolla 2000), one of the codes used in Pascucci et al. (2004). The result of the comparison with their edge-on case (inclination $i=77.5^{\circ}$ from the polar axis) is shown in the right panel of Fig. 2. Again, the agreement is satisfactory. For most wavelengths the TRADING solution is within $10 \%$ of the RADICAL solution, a difference only sligthly larger than that achieved by comparing the outputs of the various codes used in Pascucci et al. (2004). Only the high optical depth model shows a larger discrepancy on the stellar side of the SED. This is due to the limited resolution of the grid. Better agreement is obtained for the more face-on models.

As pointed out by Jonsson (2006), regular or adaptive 3D orthogonal grids are not ideally suited for a comparison with the benchmark solutions described here. In fact, no benchmark solution is available yet for the case of diffuse sources cospatial with dust, a configuration more suitable for the study of internal extinction and emission in galaxies. This is the subject of a recently started project on comparing different codes that cater to the study of radiative transfer in galaxies.

\section{The dust model}

The dust grain model of Draine \& Li (2007) has been adopted for the simulations presented in this work. The model consists in a mixture of silicate and carbonaceous grains. The size distributions was derived by fitting the extinction law and infrared emission from dust as observed in our Galaxy (Weingartner \& Draine 2001a; Li \& Draine 2001; Draine 2003a, the model for the $R_{V}=3.1$ extinction law is used here).

The dust grain extinction and absorption cross-sections have been derived using the Mie theory for spherical particles (Mie 1908; see also Bohren \& Huffman 1983). The optical properties of amorphous astronomical silicates are used for the silicate component and those of graphite for the larger carbonaceous grains.

For small carbonaceous grains, the absorption cross sections are derived from the properties of PAH materials, the most probable responsible of the MIR emission features (Li \& Draine 2001; Draine \& Li 2007). In the model used here, these grains contain $4.6 \%$ of the mass of the carbonaceous components. The properties of $\mathrm{PAH}$ grains also depend on the ionization state, and the ionization fraction of Draine \& Li (2007) is used. The absorption cross section of the carbonaceous components is assumed to change smoothly from that of PAHs to that of graphite at a radius of $0.005 \mu \mathrm{m}$ : the smooth transition provides a continuum absorption component to the PAH features for small grains, while for $a \gtrsim 0.01 \mu \mathrm{m}$ the graphite component becomes dominant (Draine \& Li 2007).

With the above recipe, I have computed the mean extinction law $A_{\lambda}$, albedo $\omega_{\lambda}$ and asymmetry parameter $g_{\lambda}$ which are used for the radiative transfer of stellar radiation $\left(A_{\lambda}\right.$ is also used for dust radiation if self-absorption is included) as well as the elements of the $Q_{j k}$ table of Eq. (13), which are necessary to compute the dust emission spectrum. In the table, $N_{\text {mat }}=4$ grain components are considered: silicate grains, with $N_{a}^{\text {sil }}=18$ bins in radius from $a=3.5 \AA$ to $0.5 \mu \mathrm{m}$; graphite grains, with $N_{a}^{\text {gra }}=10$ bins in radius from $a=0.01 \mu \mathrm{m}$ to $1 \mu \mathrm{m}$; ionised carbonaceous grains (smoothly changing from PAHs to graphite), with $N_{a}^{\mathrm{PAH}}=9$ bins in radius from $a=3.5 \AA$ to $0.01 \mu \mathrm{m}$; neutral carbonaceous grains with the same bins as for ionised grains. The values of $N_{a}^{k}$ results in similar radial bins for both carbonaceous and silicate grains. The sampling of the different components was chosen by means of trial and was dictated by the necessity of having the smallest possible number of bins (since the computing time depends on the sums in Eq. (12)) still able to provide a reasonably accurate estimate of dust emission.

Computing time is an issue especially for grains that undergoes stochastic heating. As noted by Misselt et al. (2001), this is the real bottleneck of the computation. For the models presented here, the calculation of the temperature probability distribution $P$ is limited to grains with $a \leq 0.01 \mu \mathrm{m}$ (26 bins out of 46), while the rest are assumed to emit at thermal equilibrium. The enthalpy states are sampled with $N_{T}=80$ temperature bins, with $2.7 \mathrm{~K}<T<2000 \mathrm{~K}$. The Draine \& Li (2001) models for the specific heats of bulk silicate and graphite have been used. While this temperature sampling is sufficient for the smaller grains, it does not provide correct solutions for the larger grains exposed to stronger ISRFs, whose $P$ is close to a delta function. When this happens, thermal equilibrium emission is used. A more accurate calculation would require a different temperature sampling tailored for the heating conditions in each cell and for each grain, as in the iterative algorithm described by Misselt et al. (2001). However, this is inpractical for the large grid considered here, as the method prevents the use of pre-computed tables for the terms in the transition matrix and would result in too long computation times. 


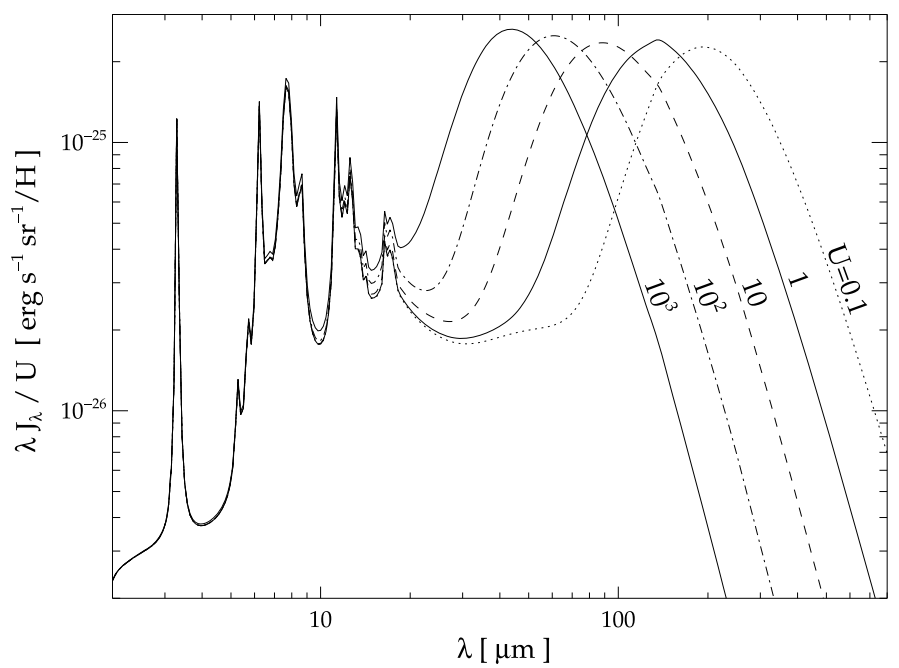

Fig. 3. Dust emission spectra for the dust model of Sect. 3, normalised to the hydrogen column density. The heating spectrum is the average Galactic ISRF, with intensity scaled by a factor $U$.

Despite the approximations and the limited grain sampling, the method used in TRADING results in a reasonably good description of dust emission. Figure 3 shows the emission when heating is due to the average Galactic ISRF (Mathis et al. 1983; see also Weingartner \& Draine 2001b), scaled by different factors $U$. In the wavelength range where emission is due to stochastically heated grains, the agreement with the analogous models of Draine \& Li (2007) is within $\approx 10 \%$. A similar agreement with full models is achieved using the spectra of Sect. 4.

The dust model is characterised by $G / D=90$ and $\tau_{V} / N_{\mathrm{H}}=$ $4.9 \times 10^{-22} \mathrm{~cm}^{2}$.

\section{An application: NGC 891}

In this section, I present TRADING models for a galactic disk. The physical quantities assumed in the simulations are tied to the case of NGC 891, an Sb-type edge-on galaxy whose SED and surface brightness distributions have been the subject of extensive studies (Xilouris et al. 1999; Popescu et al. 2000, 2004; Alton et al. 2004; Dasyra et al. 2005). In accordance with these works, a distance of $9.5 \mathrm{Mpc}$ is assumed.

For stars, I have adopted a typical spectrum of an evolved galaxy. It has been obtained by running the spectral synthesis code PEGASE.1 of Fioc \& Rocca-Volmerange (1997) with the parameters for their Sb template (see also Leitherer et al. 1996), but without including a dust correction. The intrinsic stellar spectrum is shown in Fig. 4; it is similar in shape to the average Galactic ISRF. By setting an arbitrary cut at $3 M_{\odot}$, the spectrum has been decomposed into the separate contribution of low mass stars (emitting most of $\lambda \gtrsim 0.3 \mu \mathrm{m}$ radiation, and contributing to about $80 \%$ of the bolometric luminosity), and high mass stars (emitting most of the UV radiation). This will allow one to use separate spatial distributions for young OB stars and for the older population.

In all the models, the stellar $J_{\lambda}$ is sampled with $N_{\mathrm{SED}}^{\mathrm{star}}=$ 20 bins in the range $[0.09,8] \mu \mathrm{m}$. Instead, dust emission is sampled with $N_{\text {SED }}^{\text {dust }}=150$ bins in the range $[2,1500] \mu \mathrm{m}$. For all the wavelength bins, images are produced for selected inclinations, and a global SED is obtained by integrating over the maps.

Models are compared to the SED of NGC 891, which is known over a broad wavelength range: total fluxes are available

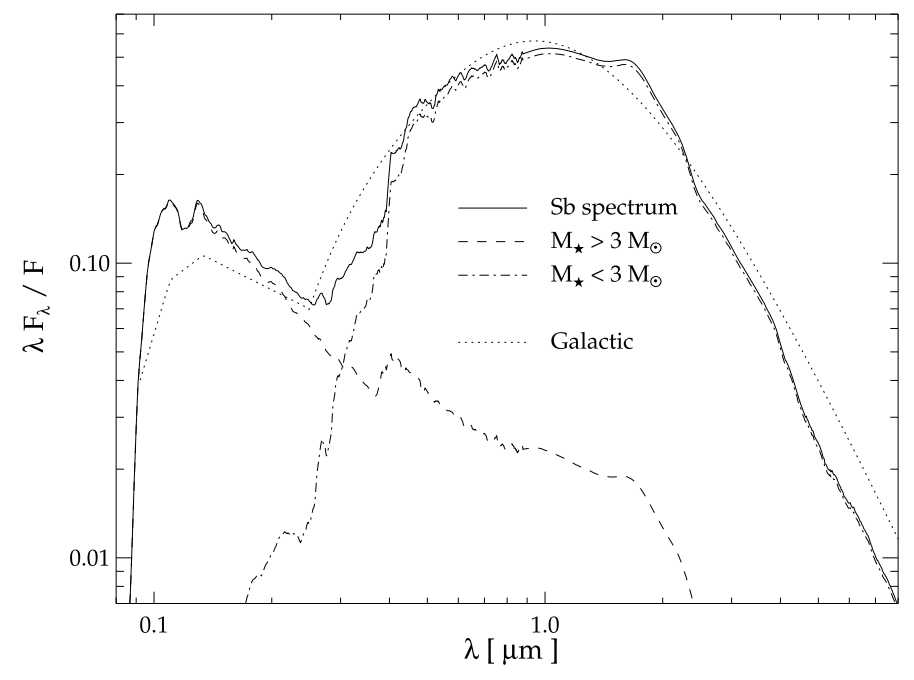

Fig. 4. The stellar SED from the $\mathrm{Sb}$ template of Fioc \& Rocca-Volmerange (1997), normalised to the bolometric luminosity. The total SED is shown together with the separate contribution of stars of low $\left(M_{\star}<3 M_{\odot}\right)$ and high $\left(M_{\star}>3 M_{\odot}\right)$ mass. The spectra have been smoothed over the wavelength. The average Galactic ISRF is also shown for comparison.

in the UV (Gil de Paz et al. 2007), optical (de Vaucouleurs et al. 1991) and NIR (Jarrett et al. 2003); dust emission has been observed at 12, 25, 60 and $100 \mu \mathrm{m}$ (Sanders et al. 2003), 170 and $200 \mu \mathrm{m}$ (Popescu et al. 2004), 260, 360 and $580 \mu \mathrm{m}$ (Dupac et al. 2003), 450 and $850 \mu \mathrm{m}$ (Alton et al. 1998) and $1.3 \mathrm{~mm}$ (Guélin et al. 1993). These fluxes are shown as datapoints in Fig. 5.

\subsection{The SED of smooth disks}

The structural properties of NGC 891 have been determined in the optical/NIR bands by Xilouris et al. (1999) (see also Xilouris et al. 1998). Surface brightness fits suggest a thin stellar disk with $h_{\mathrm{s}} / z_{\mathrm{s}} \approx 10$, and a radial scalelength $h_{\mathrm{s}}$ increasing from the $K$ to the $B$-band (a well known feature in less inclined objects; see, e.g., de Jong 1996). For simplicity, I neglect the gradient and assume $h_{\mathrm{s}}=4 \mathrm{kpc}$, a value appropriate for the NIR bands, where the bulk of stellar emission is; $z_{\mathrm{s}}=0.4 \mathrm{kpc}$ is used for the vertical scalelength.

The fits of Xilouris et al. (1999) also provide a description of the dust distribution. Since the radial scalelength of the dust disk almost doubles the NIR stellar scalelength, I have assumed $h_{\mathrm{d}}=2 h_{\mathrm{s}}$. The vertical scalelength is taken to be $z_{\mathrm{d}}=z_{\mathrm{s}} / 2$, and $\tau_{V}^{\text {f.o. }}=1$ as suggested by the same fits. Similar properties are shown by dust disks in other edge-on galaxies (Xilouris et al. 1999; Bianchi 2007).

The modelled SED are shown in Fig. 5 (left panel). The stellar radiation is assumed to have a bolometric luminosity $L_{\text {bol }}=6 \times 10^{10} L_{\odot}$, so as to provide a match of the optical/NIR observations. The result for a single stellar disk is shown for a full calculation including stochastic heating (dot-dashed line) and for a thermal equilibrium calculation using a single dust grain of mean absorption cross section (dashed line). While the simpler calculation is not able to produce significant radiation at $\lambda<40 \mu \mathrm{m}$, the output at $\lambda \gtrsim 100 \mu \mathrm{m}$ is consistent with that of the more complex case, clearly showing that most of the radiation in the dust emission peak is due to thermal equilibrium heating. It is necessary, however, to exclude from the equilibrium calculations the amount of energy (30\% for the case shown 


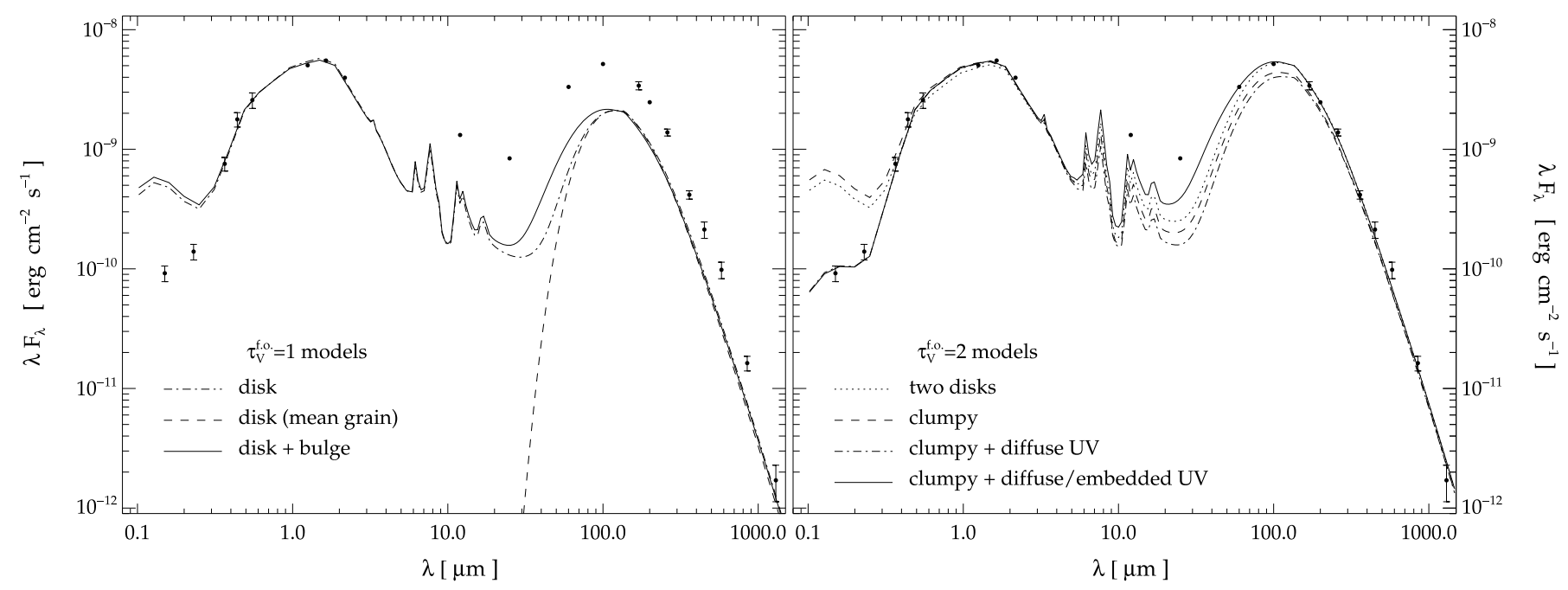

Fig. 5. SEDs for edge-on models. The left panel shows the models for a $\tau_{V}^{\text {f.o. }}=1$ and $L_{\text {bol }}=6 \times 10^{10} L_{\odot}$. As TRADING separately computes dust extinction and emission, the total SEDs have been obtained by interpolating the two outputs over the common wavelength range and summing them together. Dust emission only is shown for the case of thermal equilibrium emission by a single grain with mean properties (dashed line), after correcting for the energy absorbed by small grains. The right panel shows the SEDs of $\tau_{V}^{\text {f.o. }}=2$ models. In all cases, the luminosity emitted by low mass stars is $6 \times 10^{10} L_{\odot}$. In both panels, data points represent the observed SED of NGC 891 from the literature (see text for references). UV, optical and NIR fluxes have been corrected for foreground Galactic extinction. When not shown, error bars are smaller than symbol size.

in Fig. 5) which is absorbed by smaller grains (Bianchi et al. 2000a). In the following, all calculations will be done for the complete dust model of Sect. 3.

An important contribution to ISRF and to dust heating may come from a galactic bulge. Following the analysis of Xilouris et al. (1999), I have included a Sersic $n=4$ bulge with $b / a=0.6$ and $R_{\mathrm{e}}=1 \mathrm{kpc}$, with a luminosity $0.3 L_{\mathrm{bol}}$. The SED for this model is shown in Fig. 5 (solid line in left panel). The central concentration of the bulge results in an increased temperature for the larger grains: while in a single stellar disk model the larger carbonaceous grains attain a maximum temperature of about $28 \mathrm{~K}$ in the center ( $25 \mathrm{~K}$ for silicates), in the model including a bulge the central regions have graphite grains at $65 \mathrm{~K}$ (and silicates at $53 \mathrm{~K}$ ). This is evident in the shift of the peak of thermal emission towards shorter wavelengths. Changes are instead negligible for the stellar SED. The bulge/disk configuration will be used in the rest of the paper.

The $\tau_{V}^{\text {fo. }}=1$ disk shown so far grossly underestimates (by almost a factor two) the FIR output, as it is able to absorb (and reemit) only about $18 \%$ of the total energy. The discrepancy is the well known energy balance problem: a moderate optically thick dust disk as suggested by optical observations is not able to absorb enough energy to match the observed infrared output; absorption from a more opaque dust component is needed (Bianchi et al. 2000a; Popescu et al. 2000; Misiriotis et al. 2001). A $\tau_{V}^{\text {f.o. }}=2$ disk with $L_{\text {bol }} \approx 7.5 \times 10^{10} L_{\odot}$ (not shown) can better reproduce the dust emission, but is still $20 \%$ less bright than observations around the peak. However, such high optical depths are not not consistent with observations (Xilouris et al. 1999; Bianchi 2007).

The SEDs also fail to reproduce the MIR radiation and show an excess of UV radiation. Although the second problem can be due to an overestimate of the UV component in the intrinsic SED, it is more likely the result of an underestimate of opacity: smooth distributions for dust and stars cannot easily reproduce the localised extinction of young objects close to their dense, more opaque, birth environment.

\subsection{The SED of clumpy disks}

Since the $\tau_{V}^{\text {f.o. }}=1$ disk of Xilouris et al. (1999) fails to reproduce the FIR SED, Popescu et al. (2000) have introduced a second dust component, of mass similar to that of the first disk, but with a radial distribution equal to the stellar. This second, smooth, disk is devised to mimic the extra extinction in molecular clouds, while the first could be thought of as associated with the atomic gas. Indeed, the Interstellar Medium (ISM) in NGC 891 is made up of a broad $\mathrm{H}$ I distribution and a more centrally peaked molecular component (see, e.g., Alton et al. 2000). The distribution of molecular gas is generally found to be close to the stellar (Regan et al. 2001). The molecular and atomic gas in NGC 891 have similar masses (in total $8 \times 10^{9} M_{\odot}, 70 \%$ of which resides in a thin disk; Sofue \& Nakai 1993; Oosterloo et al. 2007), also in agreement with the mean properties of $\mathrm{Sb}$ galaxies (Young \& Scoville 1991).

In this paper, the extra mass component required to solve the energy balance problem is added in the form of a distribution of clouds. The clouds are distributed in an exponential disk of radial scalelength $h_{\mathrm{s}}$. The vertical scalelength is the same as for diffuse dust. The clumpy component has the same mass as the diffuse dust $\left(f_{\mathrm{c}}=0.5\right)$. The total dust mass in the grid is $M_{\text {dust }}=10^{8} M_{\odot}$. For the assumed $G / D$, the dust mass is roughly consistent with the gas mass derived from observations.

In the model, clouds are spherical, homogeneous and their opacity is computed from the gas surface density observed in GMC. Assuming $\Sigma=100 M_{\odot} \mathrm{pc}^{-2}$, a typical value for Local Group galaxies (Blitz et al. 2007; Rosolowsky et al. 2003), the optical depth along the diameter is $\tau_{V}^{\text {diam }} \approx 9.2$, regardless of the cloud dimensions (as follows from Eq. (8), using the dust properties of Sect. 3). According to Rosolowsky (2005), the largest clouds have a hydrogen mass $M_{\text {sup }}=10^{6.5} M_{\odot}$, which correspond to a radius $R \approx 100$ pc. GMCs in the Milky Way and a few other Local Group galaxies show a power law distribution, described by Eq. (7) with $\gamma=1.7$ (with the notable exception of M33; Blitz et al. 2007). A value of $\gamma<2$ ensures that most of the molecular mass resides in high mass clouds. 
Memory requirements may become heavy for the models of a clumpy disk, when a reasonably high resolution is required for a large number of clouds ${ }^{7}$. In particular, a lower mass limit $M_{\text {inf }}$ implies a larger number of lower mass clouds. Several tests were carried out with different values of $M_{\text {inf }}$, spanning up to two orders of magnitude in mass. The results were found to be almost independent of the choice of $M_{\text {inf }}$, because of the constant optical depth and of the luminosity of embedded radiation, which was chosen to scale with the mass of the cloud. Thus, a single cloud mass is assumed $\left(M_{\text {inf }}=M_{\text {sup }}\right)$. Because of this choice, it is possible to force subdivision down to sublevel $l=4$ inside clouds (while the adopted $E$ value ensures subdivision only down to $l=3$ ). In total, there are about 1650 clumps $^{8}$.

The case of a clumpy disk heated by diffuse radiation is shown in the right panel of Fig. 5 (dashed line). The intrinsic radiation emitted by the disk and the bulge is that for the $\mathrm{Sb}$ template of Fig. 4 , and the bolometric luminosity $L_{\mathrm{bol}}=$ $7.3 \times 10^{10} L_{\odot}$. When compared to the model in which all dust is in diffuse components (dotted line; analogous to the two dust disk model of Popescu et al. 2000), the clumpy model shows the usual reduction in the attenuation of starlight (see, e.g. Witt \& Gordon 1996; Városi \& Dwek 1999). This is reflected by a reduced dust emission in the infrared, for $\lambda \lesssim 200 \mu \mathrm{m}$. At longer wavelengths, instead, the difference between a clumpy and a smooth distribution is not appreciable. Both diffuse dust and dust in clouds externally heated by the ISRF (passive or quiescent clumps; Popescu et al. 2000) contribute to the FIR/submm emission. For the cloud optical depth considered here, dust in quiescent clumps is not substantially colder than the diffuse dust, and the spectral shape of the two components is similar. The FIR/submm emission is thus proportional to the total dust mass in a galaxy. The result holds even if $\Sigma$ (and thus the cloud optical depth) is doubled: the diffuse NIR ISRF is still able to penetrate a cloud, and the SED at longer wavelengths shows no appreciable change. Similar conclusions are drawn by Misselt et al. (2001), though they show a more pronounced dependence of the thermal peak on clumping. The difference is probably due to the different heating geometry, with a clumpy shell heated by an internal spherical distribution of stars.

Until now, all the stellar components have shared the same spectrum. In the next model, the low-mass spectrum of Fig. 4 is used for the disk and the bulge. These components have a total luminosity $6 \times 10^{10} L_{\odot}$, with $70 \%$ of the radiation emitted by the disk. This is the same luminosity the old component has in the clumpy model shown before, which is close to the observed stellar SED in the optical and NIR.

\footnotetext{
7 Executing the dust emission part of TRADING on the clumpy model presented in this section requires about $1.6 \mathrm{~Gb}$ of RAM on a 64-bit CPU to store a grid made of $3.2 \times 10^{6}$ cells $(90 \%$ of which are leaf cells $)$. The memory load is mostly due to the pointer-based structure of the binary tree. As an increase in resolution results both in an increase in memory requirements and calculation time, TRADING efficiency will certainly benefit from parallelization with a distributed memory scheme.

8 Simulations with the clumpy dust grid shown here take about $65 \mathrm{~h}$ on a 2.2 GHz AMD Athlon 64 X2 Dual Core Processor 4200+; of these, $58 \mathrm{~h}$ are needed for the dust emission calculation. Maps of $512 \times 512 \mathrm{pix}-$ els, covering the entire dust distribution, are produced for three inclinations; $N_{\text {phot }}=4 \times 10^{7}$ is sufficient for an adequate $\mathrm{S} / \mathrm{N}$ of the maps. Selfabsorption is included and iteration is stopped if the SED converges to within $5 \%$. For all the disk models presented here, only two iterations were necessary, with the SED actually converging to within a few tenths of a percent. Self-absorption attenuates dust emission in the SED for $\lambda \lesssim 10 \mu \mathrm{m}$, especially for the models seen edge-on. However, it is found to have little influence on the global energy balance.
}

Two UV emitting components are then included, sharing the high-mass spectrum of Fig. 4. The first component is a diffuse disk, with the same radial and vertical scalelengths as the clump distribution. After being extinguished by the adopted dust geometry, the spectrum becomes flat, at least for the edge-on case. If such component, with luminosity $0.8 \times 10^{10} L_{\odot}$, is added to the old stars, the stellar SED is well matched also at UV wavelengths (Fig. 5, right panel, dot-dashed line). The dust emission SED is close to that of the clumpy model with no separate emission from high-mass stars.

A second UV component is represented by point sources emitting radiation from the center of each cloud. This component of embedded sources is heavily extinguished; stellar radiation at short wavelengths is almost completely absorbed and the output spectrum peaks at $1-2 \mu \mathrm{m}$. Because of the high extinction, a considerable luminosity can be assigned to this component without modifying the stellar SED of the simulation. The luminosity is instead constrained by dust emission: the peak of thermal emission can be reproduced with $1.0 \times 10^{10} L_{\odot}$. The final SED is shown with a solid line in Fig. 5 (right panel). There is a good accordance with observations along all the spectrum, with the exception of a lower MIR flux in the simulation. I will comment on this later when discussing the dependence of the model on the chosen resolution. No significant changes in the SED can be obtained if a fraction of the cloud is taken to be only passive (e.g. without sources inside); if the UV source is positioned randomly in the cloud volume; if the UV luminosity emitted in a cloud is shared by a few sources. If instead the radiation is emitted uniformly inside the cloud, the SED peaks at colder wavelengths (an effect similar to a lack of resolution; see later). If $\Sigma$ is doubled, each cloud becomes smaller and more opaque: the SED does not change significantly, with deviations within $5 \%$ at the peak of the SED and $10 \%$ at about $20 \mu \mathrm{m}$.

In total, the model has $L_{\text {bol }}=7.8 \times 10^{10} L_{\odot}$; the UV-dominated spectrum of high-mass stars emits about $23 \%$ of $L_{\text {bol }}$ (sligthly more than in the Sb template), and $55 \%$ of this radiation is embedded inside clumps. By using standard calibrations (Kennicutt 1998), the intrinsic, dust-free, luminosity at $0.15 \mu \mathrm{m}$ can be converted to a $S F R \approx 3 M_{\odot} \mathrm{yr}^{-1}$, a value similar to the UV component used in the model of Popescu et al. (2000). Of all the bolometric radiation, 34\% is absorbed globally; $20 \%$ is absorbed at $\lambda \gtrsim 0.35 \mu \mathrm{m}$. Despite radiation from high-mass stars suffering a greater internal extinction, radiation from low-mass, older, stars still dominates the dust heating. This is in contrast with Popescu et al., where UV radiation dominates the energy balance, contributing to $70 \%$ of the FIR radiation. The reason for this discrepancy is unknown. Since the derived SFRs are similar, it may be due to model differences in the optical/NIR. In the current model, extinction of optical radiation could have been overestimated at around $\lambda=0.5 \mu \mathrm{m}$, since the stellar scalelength is taken to be about $30 \%$ shorter than that deduced from $B$ and $V$-band observations (Xilouris et al. 1999); this makes the stellar disk more concentrated with respect to the diffuse dust. However, most of observed radiation comes at longer wavelengths, where the adopted value for $h_{\mathrm{s}}$ is closer to the fits. Furthermore, the second dust disk of Popescu et al. (2000) is likely to absorb more radiation than the clumpy component in this work.

The current model also differs in the fraction of UV radiation that is embedded in clouds. In Popescu et al. (2000), dust emission caused by absorption of UV photons from localised sources is modelled using a template SED based on observations of a Galactic H II region. This template consists of a modified blackbody with emissivity $\propto \lambda^{-2}$ and $T \approx 35 \mathrm{~K}$; it does not include, in 


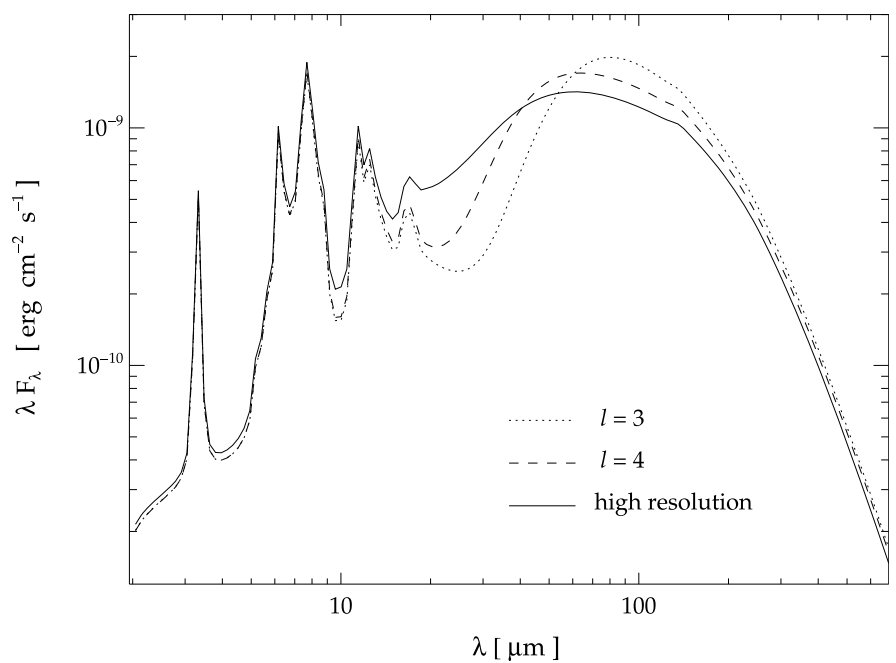

Fig. 6. Grid resolution convergence check for the emission from clouds in the clumpy model. Only dust emission is shown (see text for details).

the words of the authors, potentially cold emission components that might be expected from "parent" molecular clouds in juxtaposition to their "offspring" H II regions. From the point of view of the current work, they supply a potentially cold emission component by adding the second dust disk. However, in Popescu et al. (2000) the calculation for localised emission is not done self-consistently with that of the second, diffuse, disk. This might have altered the balance of the UV components, and be the cause of the discrepancy with the results shown here.

Dasyra et al. (2005) use the Xilouris et al. (1999) disk and increase the dust emissivity, without adding a second disk. As a result, the dust temperature reduces and the thermal emission SED shifts toward longer wavelengths, thus matching the FIR/submm observations. The missing flux at lower wavelengths is provided by absorption of UV starlight with luminosity (SFR) greater than in Popescu et al. (2000). Indeed, observations and models suggest that the dust emissivity could be greater, at least in denser environments (see, e.g., the discussion in Alton et al. 2004). However, the higher dust emissivity they derive may be simply due to the lack of cold dust emission in the $\mathrm{H}$ II region template they use (which is the same as in Popescu et al. 2000).

For the clumpy grid shown here and with the current version of TRADING, it is not possible to check for the convergence of the results by simply increasing the resolution within clouds. However, a test can be made by comparing: a simulation in which only the embedded UV radiation is emitted from the center of the clouds; a simulation of a single cloud with the high resolution grid used for the benchmark cases (Sect. 2.5), scaled to the total number of clouds. This is shown in Fig. 6 (dust emission only). If the cloud resolution is limited to sublevel $l=3$ (dotted line), the peak emission is at higher wavelengths than in the high resolution calculation (solid line). The situation improves by forcing subdivision to sublevel $l=4$ (dashed line; the value used in this paper), since higher thermal equilibrium temperatures can be sampled by a higher spatial resolution closer to the embedded source. Because of the high extinction in the UV, the central source does not significantly heat the external parts of the cloud. In full simulation these are predominantly heated by the diffuse ISRF, for which a coarser cloud and smooth medium resolution is sufficient. Thus, the differences around the peak emission are smaller than in Fig. 6. This was confirmed by tests on models with less memory requirements (smaller number of clouds). For the continuum emission at shorter wavelengths, however, differences are greater and could explain the low flux predicted at $24 \mu \mathrm{m}$ in the SEDs of Fig. 5.

Instead, the PAH features show a negligible change with resolution, since they depend only linearly, for the temperature range considered here, on the intensity of the ISRF (see, e.g., Fig. 3 and Draine \& Li 2007). The observed flux at $12 \mu \mathrm{m}$ in Fig. 5 could be matched by increasing the amount of embedded radiation by about a factor two; however, this would also shift the peak of the simulated SED to shorter wavelengths. Also, the flux cannot be increased by increasing the amount of mass in clouds (and thus their number), since the mass is constrained by the FIR/submm observations. Thus, the low predicted flux in the PAH features may point towards different ISRF intensities than can be obtained here with a simple cloud description.

The assumption of spherical, homogeneous, clouds is clearly a poor description of the complex structure of the molecular medium, which appears to be fractal (Blitz \& Williams 1999). Unfortunately, the current, non-parallel, version of TRADING and the limitations in the available computer memory do not allow a detailed study of the effects of such structure on the model output. Limited tests have been carried out by assuming that only a fraction of the cells within the spherical volume of each cloud is occupied by dust associated with molecular gas, while the remaining cells have the same properties as the underlying smooth disk. I have run two cases with filling factors $f f=0.5$ and 0.2 . In the first case, clouds maintain a structural integrity, being made up of many interconnected cells, while in the second the structure is looser (Misselt et al. 2001). The density of the dust associated with molecules increases by a factor of $1 / f f$ with respect to the standard models, and this already may pose resolution problems, expecially for the $f f=0.2$ case if the central source happens to be within a higher density cell. As expected, more UV radiation is able to escape clouds in these cases (though the $f f=0.5$ case is still consistent with observations while the $f f=0.2$ is only higher by about $30 \%$ ). As a consequence of that (and probably of the limited resolution) the flux between 20 and $80 \mu \mathrm{m}$ is lower than in the standard model, by $20 \%$ and $40 \%$ at $30 \mu \mathrm{m}$ for the $f f=0.5$ and 0.2 cases, respectively. However, there are no significant differences in the optical SED (because emission comes mainly from the diffuse medium), in the PAH range and in the thermal peak at higher wavelengths.

\subsection{Attenuation vs. inclination and images}

The global SED shown before have been obtained by integrating over simulated images for the edge-on case. However, images can be produced at any inclination. In Fig. 7, true color images are shown for the clumpy model with embedded sources, seen at $i=86^{\circ}$. The upper panel shows an image in the optical. Reddening is evident in the extinction lane and is mostly due to the diffuse disk, while clumps, due to their high optical depth, simply extinguish all radiation in their background, regardless of the wavelength. The lower panel shows an image in the FIR/submm around the peak of dust emission. Dust in clumps is hotter (bluer) in their centers, due to the embedded sources, and colder (redder) close to their surface, where the heating is mostly due to the diffuse ISRF. Emission from diffuse dust shows a gradient, which is strong only in the center of the disk, where the dust temperature is higher (mostly as an effect of the stronger ISRF in the bulge region).

The two dust disks model of Popescu et al. (2000; see also Tuffs et al. 2004) has been successful in reproducing the 

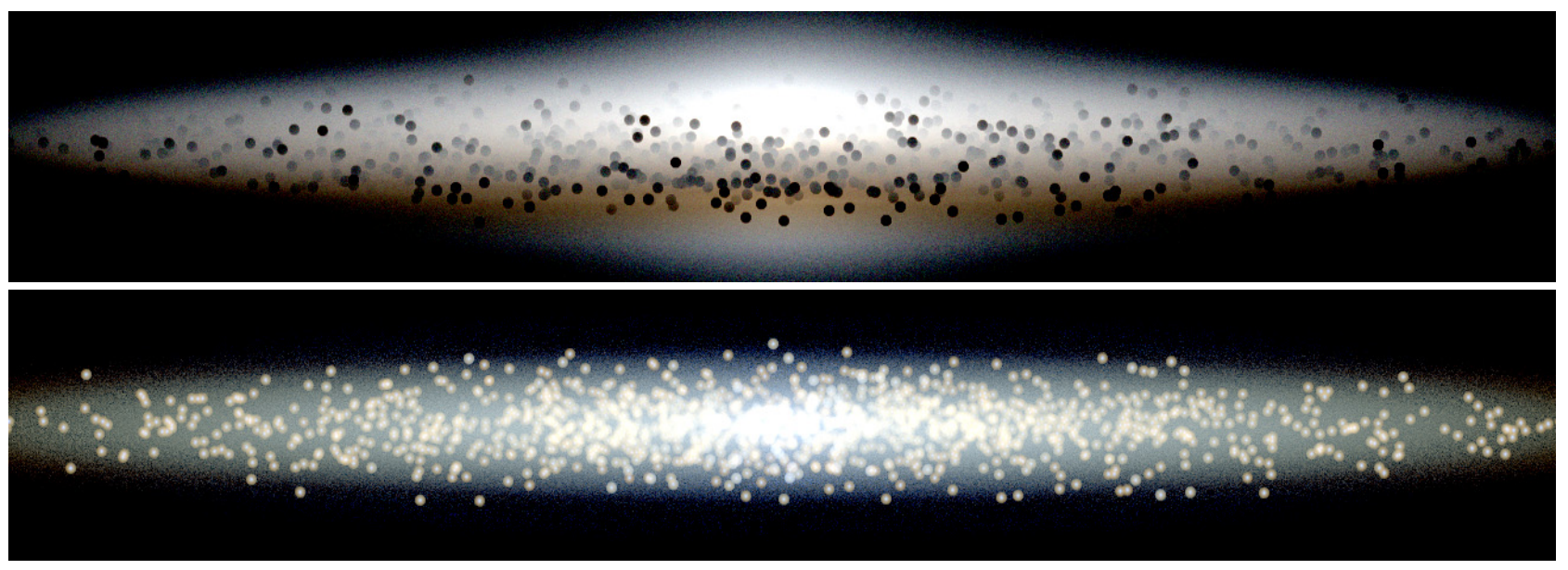

Fig. 7. RGB true color images of the clumpy model, seen from $i=86^{\circ}$. The upper panel shows an image in the optical (Red $=K$-band, Green $=V$-band, Blue $=B$-band $)$. The lower panel shows an image in the FIR/submm, around the peak of dust emission $($ Red $=850 \mu \mathrm{m}$, Green $=250 \mu \mathrm{m}$, Blue $=70 \mu \mathrm{m})$. Each panel has an extent of $32 \mathrm{kpc} \times 3.5 \mathrm{kpc}$.

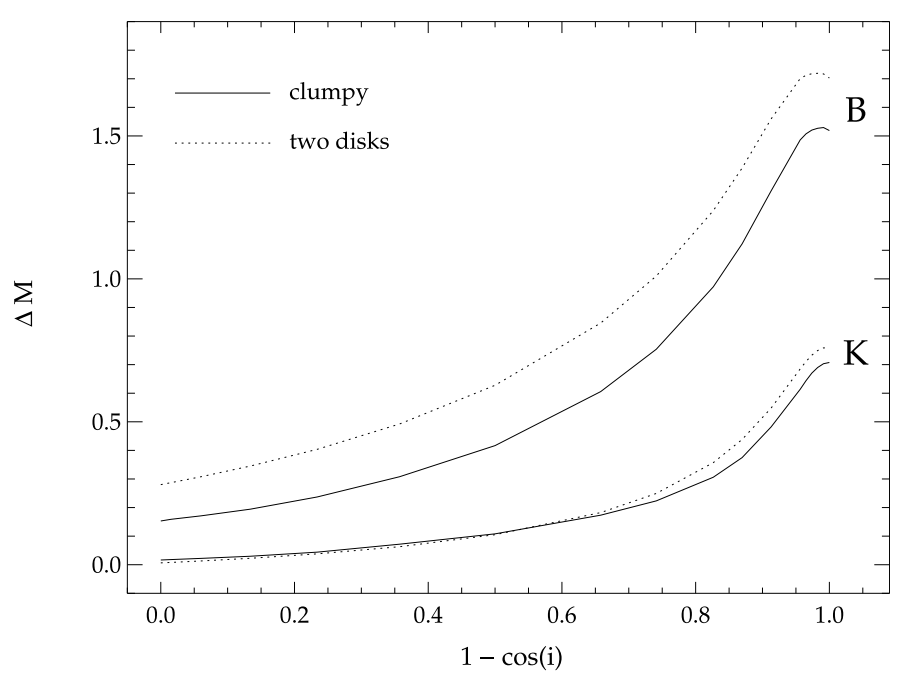

Fig. 8. Attenuation as a function of inclination. $\Delta M$ is the difference, in magnitude, between the attenuated flux and the flux that would be emitted in the absence of dust.

dependence of attenuation on inclination, as obtained from the analysis of the luminosity function of disk galaxies with different axis ratios in the Millennium Galaxy Catalogue (Driver et al. 2007). In Fig. 8, I show the difference (in magnitude) between the total magnitude and the magnitude in the no-dust case (the latter being independent of $i$ for the isotropic emission assumed here). In the $B$-band, attenuation for the clumpy model is reduced, as expected, with respect to the two disks model ${ }^{9}$. However, the dependence on $i$ is similar. Thus, the clumpy model could in principle be consistent with observations, though a more detailed study is needed, with the separate analysis of the

\footnotetext{
9 I note here that the two disks model shown in this section and in Fig. 5, though analogous in concept, is not the same as the model of Popescu et al. (2000). In this paper, it is used only to show the changes when passing from a diffuse distribution to a clumpy distribution for the dust component associated to the molecular gas. The main difference is in the radial scalelength adopted for this component. In Popescu et al. (2000) the scalelength is larger, resulting, for a similar dust mass, in a disk with a face-on optical depth roughly half of that for the molecular component in this work.
}

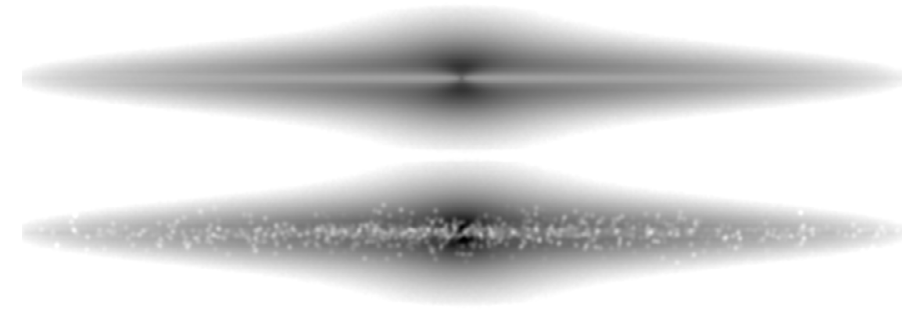

Fig. 9. $K$-band images for the two dust disks (top) and clumpy (bottom) models, seen edge-on. The extent of the images along the major axis is $32 \mathrm{kpc}$.

attenuation of the stellar bulge and disk. The reduction in attenuation (at least in the $B$-band) does not seem large enough to substantially alter the conclusions drawn by Driver et al. (2008) on the energy balance of the Cosmic SED.

In the $K$-band, the attenuation is similar both in amount and dependence on $i$. Indeed, when seen edge-on, both models show a dust lane of similar depth (Fig. 9). In the two dust disks model, most of the extinction is due to the more centrally concentrated second dust disk, which has a higher opacity $\left(\tau_{V}^{\text {f.o. }} \approx 4\right)$. In the clumpy model, it is due to clouds, which still have a non negligible optical depth $(\tau \approx 1)$ and, acting more like foreground dust, are more efficient in extinguishing radiation. Using the procedure of Bianchi (2007), the clumpy image could be fitted with a smooth disk with $\tau_{\mathrm{K}}^{\text {f.o. }} \approx 0.4$ (the fitted radial scalelength is intermediate between that of clumps and of the diffuse disk, while the vertical scalelength is retrieved correctly). This is at odds with observations, as NGC 891 and other edge-ons do not show a pronounced extinction lane and have $\tau_{\mathrm{K}}^{\text {f.o. }} \lesssim 0.1$ (Xilouris et al. 1999; Dasyra et al. 2005; Bianchi 2007). A fit to a simulated image in the $V$-band, instead, retrieves the parameter of the diffuse disk: at optical wavelengths this structure is more evident than in the NIR and coherent while the clumps behave as noisy spots that increase the fit residuals (analogous to the inhomogeneities in real images). In optical wavelengths, thus, it is possible to hide dust in clumps, though the underestimation depends on the model assumptions (Bianchi et al. 2000b; Misiriotis \& Bianchi 2002).

In Fig. 10, I show the major axis surface brightness profiles of dust emission for the two disks and the clumpy models 


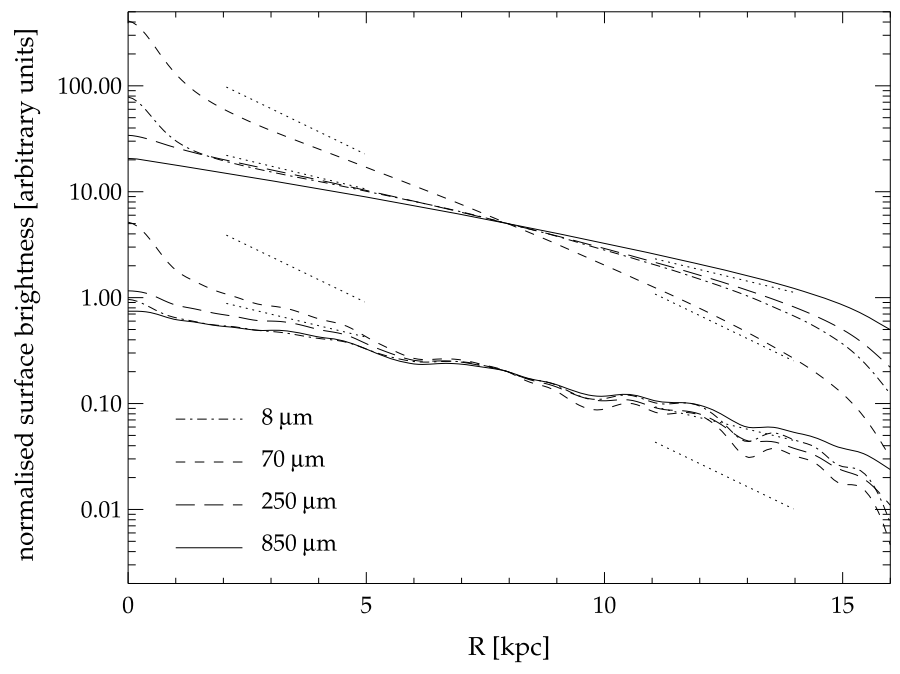

Fig. 10. Major axis surface brightness profiles for the two disks (top) and clumpy (bottom) models, seen edge-on. The profiles have been convolved with a Gaussian PSF $\left(F W H M=16^{\prime \prime}\right)$, smoothed by folding the left and right part of the image (for the clumpy model) and arbitrarily normalised at $8 \mathrm{kpc}$. As an aid to the eye, the dotted segments show the slopes of exponential fall-offs with scalelengths $h_{\mathrm{s}}=4 \mathrm{kpc}$ and $h_{\mathrm{s}} / 2$.

seen edge-on. Images have been smoothed with a PSF of $F W H M=16^{\prime \prime}$, a resolution similar to what can be achieved, currently or in the next few years, at $70 \mu \mathrm{m}$ using MIPS aboard the satellite Spitzer (Rieke et al. 2004), at $250 \mu \mathrm{m}$ using SPIRE aboard the satellite Herschel (Griffin et al. 2007) and at $850 \mu \mathrm{m}$ using the submm cameras SCUBA2 (Holland et al. 2006) and LABOCA (Kreysa et al. 2003). To these, I have added a profile at about $8 \mu \mathrm{m}$, centered on a large PAH feature. For a diffuse dust disk co-spatial with the heating sources, as is the case in the two dust disk model for the second dust disk and the stellar disk, the emission gradient becomes less steep as the wavelength increases. In the submm, the Rayleigh-Jeans spectrum does not depend strongly on the dust temperature, and the gradient approaches the intrinsic slope of the dust distribution (close to the radial scalelength $h_{\mathrm{s}}{ }^{10}$ ). Instead, at wavelengths shorter than the peak, the spectrum depends more on the strength of the ISRF, as shown by the $70 \mu \mathrm{m}$ profile (the further steepening in the center being due to the bulge). This is not the case for the PAH emission, however, because of the linear dependence of the spectrum on the ISRF intensity.

When the dust mass of the second disk is distributed in clumps (retaining the same radial scalelength), the differences between the profiles at all wavelengths are reduced. This is caused mainly by the contribution of internal clump emission at $\lambda<200 \mu \mathrm{m}$, which is independent of the clump position. Also, the $8 \mu \mathrm{m}$ profile becomes closer to the submm, explaining the observed correlation between PAHs and cold dust (see, e.g. Haas et al. 2002). Though reduced, the bulge contribution to the $70 \mu \mathrm{m}$ emission is still detectable (see also Fig. 7).

\footnotetext{
10 The radial profile at $850 \mu \mathrm{m}$ is steeper than the exponential of scalelength $h_{\mathrm{s}}$, both because of the contribution of the diffuse disk, and because the projected profile of an exponential disk seen edge-on is $\propto r K_{1}(r / h)$, with $K_{1}(x)$ the modified Bessel function of the second kind and first order and $r$ the distance from the center (Kylafis \& Bahcall 1987). For an infinite disk, the profile tends to the exponential. In the case shown here, the second dust disk, the distribution of clumps, and the stellar disk are all truncated at $4 h_{\mathrm{s}}$. This causes the profile steepening seen at $16 \mathrm{kpc}$. The truncation has little effect on the results.
}

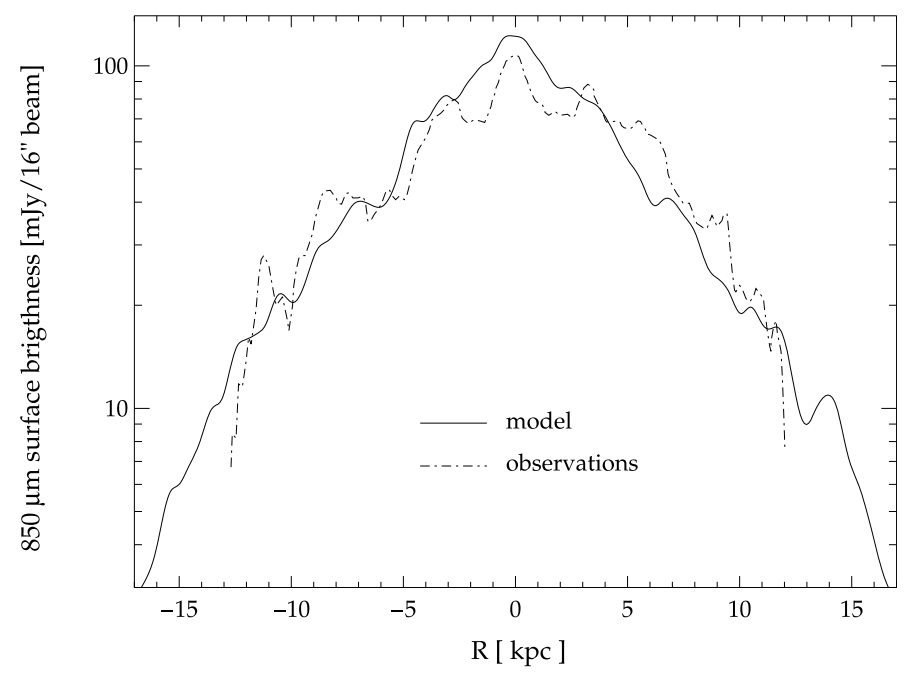

Fig. 11. Major axis surface brightness profile at $850 \mu \mathrm{m}$ for the clumpy model, compared to the SCUBA observations of Alton et al. (2000). The model has been convolved to the SCUBA beam $\left(F W H M=16^{\prime \prime}\right)$ and the profile smoothed as in Alton et al. (2000).

Finally, I show in Fig. 11 a comparison between the clumpy model and the SCUBA major axis profile as presented in Alton et al. (2000). As for the model of Popescu et al. (2000, see their Fig. 6) there is a broad agreement with observations, both in flux level and gradient. Only, the clumpy model appears to be smoother than observations, possibly hinting at a more complex structure in the cloud distribution than what is adopted for this paper.

\section{Summary}

I have presented in this paper the Monte Carlo radiative transfer code TRADING. It is designed to study continuum extinction and emission in a dusty medium. The main features of TRADING are:

i. A description of the dust distribution based on a binary-tree adaptive grid.

ii. The possibility of emitting stellar radiation from several diffuse distributions and collections of point sources/clouds, each with an independent spectrum.

iii. Dust emission from a distribution of grains of different sizes and materials, under thermal equilibrium or stochastic heating conditions.

iv. Self absorption through an iterative process.

The code shows an excellent agreement with benchmark cases and can be easily adapted to several environments of astrophysical interest. For the application shown in this paper, TRADING has been tailored to the case of a galactic disk. The disk includes: a diffuse dust component; a clumpy component made of a distribution of spherical clouds, with properties modelled on those of the GMCs in local galaxies. Building on the studies of Xilouris et al. (1999) and Popescu et al. (2000), simulations have been produced for the well known case of the edge-on spiral NGC 891. The main results can be summarised as follows.

1. The global SED of NGC 891 from the UV to the mm can be reproduced by a model including: a diffuse dust disk, more widespread than the stellar and of moderate optical 
depth $\left(\tau_{V}^{\text {f.o. }}=1\right)$; a clumpy dust distribution, of mass similar to that of the diffuse disk; a population of old stars, smoothly distributed in an exponential disk and a bulge, emitting about $75 \%$ of the bolometric radiation; a population of young stars emitting the remaining fraction mainly in the UV, roughly half of which is embedded in clouds. Despite the increased attenuation for UV radiation, the old stars are the main contributor to dust heating (about $60 \%$ of all radiation emitted by dust). This is in contrast with the result of Popescu et al. (2000), which predicts a dominant UV contribution to heating.

In the MIR, the current model produces less radiation than the observations. This can be only in part explained with a lack of spatial resolution for clouds. The difference possibly can be due to a more complex structure of the ISM and ISRF than modelled here. This could also be the reason for the greater extinction in the $K$-band image and the smoother appearence of the $850 \mu \mathrm{m}$ than observed.

2. Dust in clouds is found to be significantly heated by both the embedded UV radiation and the NIR radiation from the diffuse stellar distribution penetrating the outer shells of a cloud. This second source of heating results in cold dust emission which is not present in the template for localised emission used by Popescu et al. (2000) and Dasyra et al. (2005). In Popescu et al. (2000), cold dust emission is provided by a second diffuse dust disk; however, contrary to this work, the radiative transfer calculation for the extra dust component in Popescu et al. (2000) is not done self-consistently with that for localised sources. This is the cause of the larger fraction of embedded UV radiation in the current work. Also, the difference between a clumpy and smooth disk results in a smaller attenuation of diffuse radiation at optical wavelengths in the current model. In Dasyra et al. (2005), the lack of cold dust emission associated with localised emission appears to be the main reason for their claim for a higher dust emissivity in the FIR. Other models which compute the contribution of clouds independently from that of the diffuse ISM, such as GRASIL (Silva et al. 1998), might need to include external heating of clouds by a diffuse ISRF. Because of the diffuse heating, cold emission in the submm comes from all the dust components.

3. In a clumpy disk, and for the resolution of current and future instruments, color gradients are rather similar along all the dust emission spectrum. They follow the gradients of stars and of the main dust component. A steepening may be observed in the Wien side of the spectrum for thermal equilibrium heated grains $(20 \mu \mathrm{m} \lesssim \lambda \lesssim 100 \mu \mathrm{m})$, mostly because of the presence of a bulge.

The conclusions drawn on the energy budget for the single object NGC 891 might not be of general application for other galaxies, though NGC 891 is considered to be a prototypical spiral. Unfortunately, not many galaxies have detailed observations of the FIR/submm beyond the thermal peak. However, the advent of the SPIRE instrument aboard the Herschel satellite (Griffin et al. 2007), in conjunction with the new ground-based submm facilities, will soon permit a more detailed study of this spectral range, necessary for constraining the bulk of dust mass.

Acknowledgements. I am grateful to Maarten Baes, Bruce Draine, Edvige Corbelli, Karl Gordon, Cristina Popescu, Malcolm Walmsley and Emmanuel Xilouris for helpful suggestions and discussions. I thank the anonymous referee for constructive comments that improved the contents and presentation of this paper. This research has made use of the NASA/IPAC Extragalactic Database (NED) which is operated by the Jet Propulsion Laboratory, California
Institute of Technology, under contract with the National Aeronautics and Space Administration. This research has made use of NASA's Astrophysics Data System. This paper is dedicated to the memory of Angelos Misiriotis, a fond radiative transfer friend who passed away too prematurely.

\section{References}

Alton, P. B., Bianchi, S., Rand, R. J., et al. 1998, ApJ, 507, L125

Alton, P. B., Xilouris, E. M., Bianchi, S., et al. 2000, A\&A, 356, 795

Alton, P. B., Xilouris, E. M., Misiriotis, A., Dasyra, K. M., \& Dumke, M. 2004, A\&A, 425, 109

Baes, M., \& Dejonghe, H. 2001, MNRAS, 326, 722

Baes, M., Davies, J. I., Dejonghe, H., et al. 2003, MNRAS, 1081

Baes, M., Dejonghe, H., \& Davies, J. I. 2004, in Dark Matter in Galaxies, ed.

S. Ryder, D. Pisano, M. Walker, \& K. Freeman, IAU Symp., 220, 343

Baes, M., Stamatellos, D., Davies, J. I., et al. 2005, New Astron., 10, 523

Bianchi, S. 2007, A\&A, 471, 765

Bianchi, S., Ferrara, A., \& Giovanardi, C. 1996, ApJ, 465, 127

Bianchi, S., Davies, J. I., \& Alton, P. B. 2000a, A\&A, 359, 65

Bianchi, S., Ferrara, A., Davies, J. I., \& Alton, P. B. 2000b, MNRAS, 311, 601

Bjorkman, J. E., \& Wood, K. 2001, ApJ, 554, 615

Blitz, L., \& Williams, J. P. 1999, in the Origin of Stars and Planetary Systems, NATO ASIC Proc., 540, 3

Blitz, L., Fukui, Y., Kawamura, A., et al. 2007, in Protostars and Planets V, ed. B. Reipurth, D. Jewitt, \& K. Keil, 81

Bohren, C. F., \& Huffman, D. R. 1983, Absorption and Scattering of Light by Small Particles (New York: Wiley)

Cashwell, E. D., \& Everett, C. J. 1959, A Practical Manual on the Monte Carlo Method for Random Walk Problems (New York: Pergamos)

Dasyra, K. M., Xilouris, E. M., Misiriotis, A., \& Kylafis, N. D. 2005, A\&A, 437, 447

de Jong, R. 1996, A\&A, 313, 377

de Vaucouleurs, G. 1959, Handbuck der Physik, ed. S. Flugge (Berlin: Springer), 53,275

de Vaucouleurs, G., de Vaucouleurs, A., Corwin, Herold, G. J., et al. 1991, Third Reference Catalogue of Bright Galaxies (Berlin: Cambridge University Press), RC3

Draine, B. T. 2003a, ARA\&A, 41, 241

Draine, B. T. 2003b, ApJ, 598, 1017

Draine, B. T., \& Li, A. 2001, ApJ, 551, 807

Draine, B. T., \& Li, A. 2007, ApJ, 657, 810

Driver, S. P., Popescu, C. C., Tuffs, R. J., et al. 2007, MNRAS, 379, 1022

Driver, S. P., Popescu, C. C., Tuffs, R. J., et al. 2008, ApJ, L803

Dullemond, C. P., \& Turolla, R. 2000, A\&A, 360, 1187

Dupac, X., del Burgo, C., Bernard, J., et al. 2003, MNRAS, 344, 105

Fioc, M., \& Rocca-Volmerange, B. 1997, A\&A, 326, 950

Frisken, S. F., \& Perry, R. 2002, J. Graphics Tools, 7, 1

Gil de Paz, A., Boissier, S., Madore, B. F., et al. 2007, ApJS, 173, 185

Gordon, K. D., Misselt, K. A., Witt, A. N., \& Clayton, G. C. 2001, ApJ, 551, 269

Griffin, M., Abergel, A., Ade, P., et al. 2007, Adv. Sp. Res., 40, 612

Guélin, M., Zylka, R., Mezger, P. G., et al. 1993, A\&A, 279, L37

Guhathakurta, P., \& Draine, B. T. 1989, ApJ, 345, 230

Haas, M., Klaas, U., \& Bianchi, S. 2002, A\&A, 385, L23

Henyey, L. G., \& Greenstein, J. L. 1941, ApJ, 93, 70

Holland, W., MacIntosh, M., Fairley, A., et al. 2006, in Millimeter and

Submillimeter Detectors and Instrumentation for Astronomy III., ed.

J. Zmuidzinas, W. S. Holland, S. Withington \& W. D. Duncan, Proc. SPIE, $6275,62751 \mathrm{E}$

Holwerda, B. W., Keel, W. C., \& Bolton, A. 2007, AJ, 134, 2385

Ivezic, Z., \& Elitzur, M. 1997, MNRAS, 287, 799

Ivezic, Z., Groenewegen, M. A. T., Men'shchikov, A., \& Szczerba, R. 1997, MNRAS, 291, 121

Jarrett, T. H., Chester, T., Cutri, R., Schneider, S. E., \& Huchra, J. P. 2003, AJ, 125,525

Jonsson, P. 2006, MNRAS, 372, 2

Juvela, M. 2005, A\&A, 440, 531

Kennicutt, R. C. 1998, ARA\&A, 36, 189

Kreysa, E., Bertoldi, F., Gemuend, H.-P., et al. 2003, in Millimeter and Submillimeter Detectors for Astronomy, ed. T. G. Phillips, \& J. Zmuidzinas, Proc. SPIE, 4855, 41

Kurosawa, R., \& Hillier, D. J. 2001, A\&A, 379, 336

Kylafis, N. D., \& Bahcall, J. N. 1987, ApJ, 317, 637

Leitherer, C., Alloin, D., Fritz, V., Alvensleben, U., et al. 1996, PASP, 108, 996

Li, A., \& Draine, B. T. 2001, ApJ, 554, 778

Li, Y., Hopkins, P. F., Hernquist, L., et al. 2008, ApJ, 678, 41 
Lucy, L. B. 1999, A\&A, 344, 282

Mathis, J. S., Mezger, P. G., \& Panagia, N. 1983, A\&A, 128, 212

Matthews, L. D., \& Wood, K. 2001, ApJ, 548, 150

Mie, G. 1908, Ann. Phys., 25, 377

Misiriotis, A., \& Bianchi, S. 2002, A\&A, 384, 866

Misiriotis, A., Popescu, C. C., Tuffs, R., \& Kylafis, N. D. 2001, A\&A, 372, 775

Misselt, K. A., Gordon, K. D., Clayton, G. C., \& Wolff, M. J. 2001, ApJ, 551, 277

Niccolini, G., Woitke, P., \& Lopez, B. 2003, A\&A, 399, 703

Oosterloo, T., Fraternali, F., \& Sancisi, R. 2007, AJ, 134, 1019

Pascucci, I., Wolf, S., Steinacker, J., et al. 2004, A\&A, 417, 793

Pierini, D., Gordon, K. D., Witt, A. N., \& Madsen, G. J. 2004, ApJ, 617, 1022

Popescu, C. C., Misiriotis, A., Kylafis, N. D., Tuffs, R. J., \& Fischera, J. 2000, A\&A, 362, 138

Popescu, C. C., Tuffs, R. J., Kylafis, N. D., \& Madore, B. F. 2004, A\&A, 414, 45 Prugniel, P., \& Simien, F. 1997, A\&A, 321, 111

Regan, M. W., Thornley, M. D., Helfer, T. T., et al. 2001, ApJ, 561, 218

Rieke, G. H., Young, E. T., Engelbracht, C. W., et al. 2004, ApJS, 154, 25

Rocha, M., Jonsson, P., Primack, J. R., \& Cox, T. J. 2008, MNRAS, 383, 1281

Rosolowsky, E. 2005, PASP, 117, 1403

Rosolowsky, E., Engargiola, G., Plambeck, R., \& Blitz, L. 2003, ApJ, 599, 258
Sanders, D. B., Mazzarella, J. M., Kim, D.-C., Surace, J. A., \& Soifer, B. T. 2003, AJ, 126, 1607

Sersic, J. L. 1968, Atlas de galaxias australes (Cordoba, Argentina: Observatorio Astronomico)

Siewert, C. E., \& Maiorino, J. R. 1979, J. Quant. Spec. Radiat. Transf., 22, 435

Silva, L., Granato, G. L., Bressan, A., \& Danese, L. 1998, ApJ, 509, 103

Sofue, Y., \& Nakai, N. 1993, PASJ, 45, 139

Tuffs, R. J., Popescu, C. C., Völk, H. J., Kylafis, N. D., \& Dopita, M. A. 2004, A\&A, 419, 821

Városi, F., \& Dwek, E. 1999, ApJ, 523, 265

Weingartner, J. C., \& Draine, B. T. 2001a, ApJ, 548, 296

Weingartner, J. C., \& Draine, B. T. 2001b, ApJS, 134, 263

Witt, A. N., \& Gordon, K. D. 1996, ApJ, 463, 681

Wolf, S. 2003, ApJ, 582, 859

Wolf, S., Henning, T., \& Stecklum, B. 1999, A\&A, 349, 839

Xilouris, E. M., Alton, P. B., Davies, J. I., et al. 1998, A\&A, 331, 894

Xilouris, E. M., Byun, Y. I., Kylafis, N. D., Paleologou, E. V., \& Papamastorakis, J. 1999, A\&A, 344, 868

Young, J. S., \& Scoville, N. Z. 1991, ARA\&A, 29, 581

Yusef-Zadeh, F., Morris, M., \& White, R. L. 1984, ApJ, 278, 186 